\title{
Intrinsic 4-1BB signals are indispensable for the establishment of an influenza-specific tissue-resident memory CD8 T-cell population in the lung
}

\author{
AC Zhou ${ }^{1}$, LE Wagar ${ }^{1,2}$, ME Wortzman ${ }^{1,2}$ and TH Watts ${ }^{1}$
}

The induction of long-lived heterotypic T-cell protection against influenza virus remains elusive, despite the conservation of T-cell epitopes. T-cell protection against influenza is critically dependent on lung-resident memory $\mathrm{T}$ cells (Trm). Here we show that intranasal administration of 4-1BBL along with influenza nucleoprotein in a replicationdefective adenovirus vector to influenza pre-immune mice induces a remarkably stable circulating effector memory CD8 T-cell population characterized by higher IL-7R $\alpha$ expression than control-boosted T cells, as well as a substantial lung parenchymal $\mathrm{CD}_{69}{ }^{+} \mathrm{CD} 8 \mathrm{Trm}$ population, including both $\mathrm{CD}_{103^{+}}$and $\mathrm{CD} 103^{-}$cells. These T-cell responses persist to greater than 200 days post-boost and protect against lethal influenza challenge in aged (year old) mice. The expansion of the nucleoprotein-specific CD8 Trm population during boosting involves recruitment of circulating antigen-specific cells and is critically dependent on local rather than systemic administration of 4-1BBL as well as on 4-1BB on the CD8 Tcells. Moreover, during primary influenza infection of mixed bone marrow chimeras, 4-1BB-deficient Tcells fail to contribute to the lung-resident Trm population. These findings establish both endogenous and supraphysiological 4-1BBL as a critical regulator of lung-resident memory CD8 T cells during influenza infection.

\section{INTRODUCTION}

A universal vaccine against influenza virus remains elusive. Although the induction of broadly neutralizing antibodies to influenza virus shows promise, ${ }^{1}$ cross-protective T-cell immunity will likely be an important component of any broadly protective long-lasting vaccine. T-cell epitopes are highly conserved in diverse influenza strains. ${ }^{2}$ However, even a live influenza infection only transiently boosts circulating T-cell responses in previously immune adults. ${ }^{3,4}$ Moreover, older people have impaired immunity to influenza and are highly vulnerable to influenza-induced morbidity and mortality. ${ }^{5}$ Therefore, we need a means of inducing long-lived protective immunity that could be administered in midlife to allow protection late in life.

Tissue-resident memory $\mathrm{T}$ cells ( $\mathrm{Trm}$ ) reside in tissue to provide a first line of defense against recurrent infection. ${ }^{6,7} \mathrm{Trm}$ are induced when $\mathrm{T}$ cells enter the infected tissue during primary infection and differentiate in situ to form a non- circulating population that can persist to rapidly respond upon re-exposure to antigen. ${ }^{6,7}$ Within the lung, CD8 Trm have been shown to mediate protective heterotypic immunity to influenza infection. ${ }^{8,9}$ However, longevity is a problem as lung CD8 Trm disappear within months after influenza infection, and heterotypic immunity is lost despite steady numbers of memory CD8 T cells in the spleen and circulation that can still traffic into the lung tissue. ${ }^{8,10,11}$

CD137 (4-1BB) signals are particularly potent in enhancing the expansion and maintenance of CD8 effector and memory $\mathrm{T}$ cells. ${ }^{12}$ Moreover, lack of 4-1BBL impairs the recall response to influenza virus, ${ }^{13}$ attributed to a loss of effector memory T cells at the late stage of the primary response. ${ }^{14}$ However, to date, the effect of 4-1BBL on tissue-resident memory $\mathrm{T}$ cells has not been examined.

Most individuals receive influenza vaccines in adulthood after previous exposure to natural influenza infection. Therefore, to mimic the effect of vaccination of a previously immune

${ }^{1}$ Department of Immunology, Faculty of Medicine, University of Toronto, Toronto, Ontario, Canada. Correspondence: TH Watts (tania.watts@utoronto.ca) ${ }^{2}$ Present addresses: L.E.W. Department of Microbiology and Immunology, Stanford University, Stanford, CA 94305-5323, USA; M.E.W. Canadian Cancer Society Research Institute, Toronto, ON M4V 2Y7, Canada. 
adult, we asked whether boosting with influenza nucleoprotein (NP) together with 4-1BBL improves the duration of protection to influenza virus. We show that intranasal (i.n.) immunization of influenza-immune mice with a replication-defective adenovirus containing influenza $\mathrm{NP}$ and $4-1 \mathrm{BBL}$ results in induction of a long-lived circulating memory population of effector memory T cells (Tem) as well as a substantial Trm population in the lung that protects mice against lethal heterotypic influenza challenge into old age (1-year-old). These effects require 4-1BB on the CD8 T cells. Remarkably, in mixed bone marrow chimeras 4-1BB-deficient $\mathrm{T}$ cells are almost completely absent from the lung parenchymal pool of memory CD8 $\mathrm{T}$ cells established during primary influenza infection. These findings establish an essential role for 4-1BB/41BBL interactions in the formation of persistent CD8 Trm within the lung during influenza infection.

\section{RESULTS}

Local stimulation of 4-1BB in a secondary immunization augments the number of circulating memory CD8 T cells and provides long-lived protection against lethal influenza challenge

Intranasal administration of a replication-defective adenovirus containing the influenza NP gene and 4-1BBL (Ad-NP-4$1 \mathrm{BBL}$ ) can lower the required vaccine dose for induction of $\mathrm{NP}_{366-374}$-specific CD8 $\mathrm{T}$ cells that is protective against lethal influenza challenge compared with a vaccine containing NP (Ad-NP) alone. ${ }^{15}$ However, this effect is short lived, providing protection for only an additional 3 months over NP alone. ${ }^{15}$ Moreover, studies of T-cell responses during the 2009 pandemic showed that even a live infection induces only transient boosting of circulating pre-existing CD8 memory populations in humans. ${ }^{3,4}$ Thus, there is a need to improve upon even the natural infection to obtain long-term robust T-cell immunity to influenza virus. As $4-1 \mathrm{BB}$ stimulation is more effective in a secondary as compared with a primary CD8 T-cell response, ${ }^{16-18}$ we asked whether delivery of 4-1BBL in a replication-defective adenovirus vaccine to influenza-immune mice would induce longer-lived protection against lethal influenza infection. Accordingly, mice were infected with a low dose of influenza virus $\mathrm{A} / \mathrm{HK} / \mathrm{X} 31(\mathrm{X} 31)^{19}$ and then either left unboosted or boosted 1 month later with replicationdefective adenovirus alone (control Ad), Ad-NP, or Ad-NP-4$1 \mathrm{BBL}$ (Figure 1a). We chose a vector dose of $10^{6} \mathrm{pfu}$ per mouse, which is 100 -fold lower than the optimum dose required for maximal $\mathrm{NP}_{366-374}$-specific $\mathrm{T}$-cell expansion with Ad-NP alone during priming. ${ }^{15}$ Circulating memory $\mathrm{CD} 8 \mathrm{~T}$ cells were followed in the blood for 9-11 months in two separate cohorts of mice, followed by influenza challenge. Inclusion of 4-1BBL in the vector dramatically enhanced the expansion of circulating $\mathrm{NP}_{366-374}$-specific CD8 $\mathrm{T}$ cells, which reached a maximum of $30 \%$ of circulating CD8 $\mathrm{T}$ cells by day 7 , before declining over the next few weeks, and stabilizing at $\sim 10 \%$ of the CD8 T-cell population between day 60 and 120 (Figure 1b,c). Remarkably, this stable population of circulating CD8 memory cells persisted to at least 300 days. Similar results were recapitulated in the spleen for at least 7 months, where there was not only a higher frequency of $\mathrm{NP}_{366-374}$-specific memory cells in the circulating CD8 T-cell pool, but also an increase in the absolute number in Ad-NP-4-1BBL compared with Ad-NP or control-boosted groups (Figure 1d). At this low vector dose, the response to Ad-NP was only marginally higher than that of control Ad-boosted or unboosted mice (Figure 1b).

To test the protective efficacy of Ad-NP-4-1BBL treatment, mice were challenged with a lethal dose of influenza A/PR8 (PR8) at 9-11 months post-boost. PR8 shares the same NP epitope as the initial priming X31 strain but contains different $\mathrm{HA}$ and NA proteins (PR8 is H1N1, whereas $\mathrm{X} 31$ is $\mathrm{H} 3 \mathrm{~N} 2$ ), thereby allowing us to assess protection from CD8 T-cell immunity without the interference of neutralizing antibody responses. ${ }^{19}$ Mice pre-treated with Ad-NP-4-1BBL were protected from influenza-mediated weight loss and the majority survived challenge, whereas Ad-NP boosting offered a much smaller survival advantage over controls (Figure 1e,f). There were no signs of splenomegaly or liver toxicity as measured by alanine transaminase levels in the serum (data not shown), suggesting that the localized 4-1BBL administration avoids the pathological effects that have been associated with systemic administration of anti-4-1BB agonistic antibody. ${ }^{20}$

\section{4-1BBL-induced circulating memory cells have characteristics of long-lived effectors}

Circulating memory $\mathrm{T}$ cells can be characterized as central memory $(\mathrm{Tcm})$ or effector memory $(\mathrm{Tem})$ based on their migration patterns and functional properties. Tcm are identified by high expression of CD62L and CCR7, and are more quiescent with greater proliferative potential upon

\footnotetext{
Figure 1 Intranasal (i.n.) delivery of 4-1BBL in a replication-defective adenovirus vector with influenza NP induces a long-lived circulating memory CD8 T-cell population and protects against lethal flu challenge. (a) Mice were infected i.n. with A/HK/X31 (5 HAU/mouse) and then left unboosted or boosted i.n. 1 month later with a control replication-deficient adenovirus (control Ad), adenovirus containing the influenza NP gene (Ad-NP), or adenovirus containing NP and 4-1BBL (Ad-NP-4-1BBL) at $10^{6}$ pfu per mouse. (b) Influenza NP ${ }_{366-374}$-specific CD8 T cells were tracked in the blood using the $\mathrm{D}^{\mathrm{b}}$ / $\mathrm{NP}_{366-374}$ tetramer, with (c) representative gating at 10 months (day 300 ) post-boost. Control here and in subsequent figures refers to pooled data from unboosted and control Ad boosted, which gave indistinguishable results. Statistical analysis is shown between Ad-NP and Ad-NP-4-1BBL-boosted groups. (d) Frequency (of total CD8 T cells) and absolute numbers of $\mathrm{D}^{\mathrm{b}} / \mathrm{NP}_{366-374}$ tetramer ${ }^{+} \mathrm{CD} 8 \mathrm{~T}$ cells quantified in the spleen at 7 months (day 210 ) post-boost. Mice were challenged at 9-11 months (day 270-330) post-boost with a lethal dose of A/PR8 influenza $\left(5 \times 10^{7} \mathrm{TCID}_{50} / \mathrm{mouse}\right)$. (e) Weight loss and (f) survival curve are shown post-challenge. Data in $\mathbf{b}, \mathbf{c}$, and f) are pooled from two separate experiments with $n=6-8$ per group per experiment, whereas (e) shows a representative of the two experiments (mean \pm s.e.m.). (d) was pooled from two separate experiments with $n=4$ per group per experiment. ${ }^{\star \star} P<0.005,{ }^{\star \star \star} P<0.0005,{ }^{\star * \star \star} P<0.00005$ (two-way ANOVA test (b,e), Kruskal-Wallis test (d), or Mantel-Cox test (f)).
} 
a

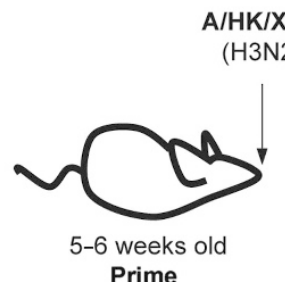
Prime

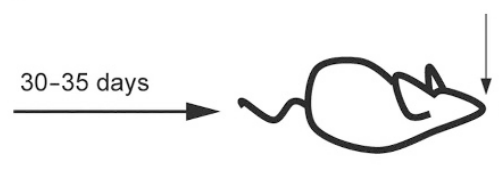

Boost

Unboosted or

Control Ad or

Ad-NP or

A/PR8

Ad-NP-4-1BBL

b

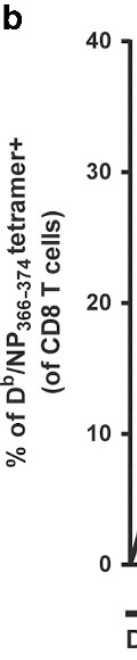

Long-term tracking - Blood

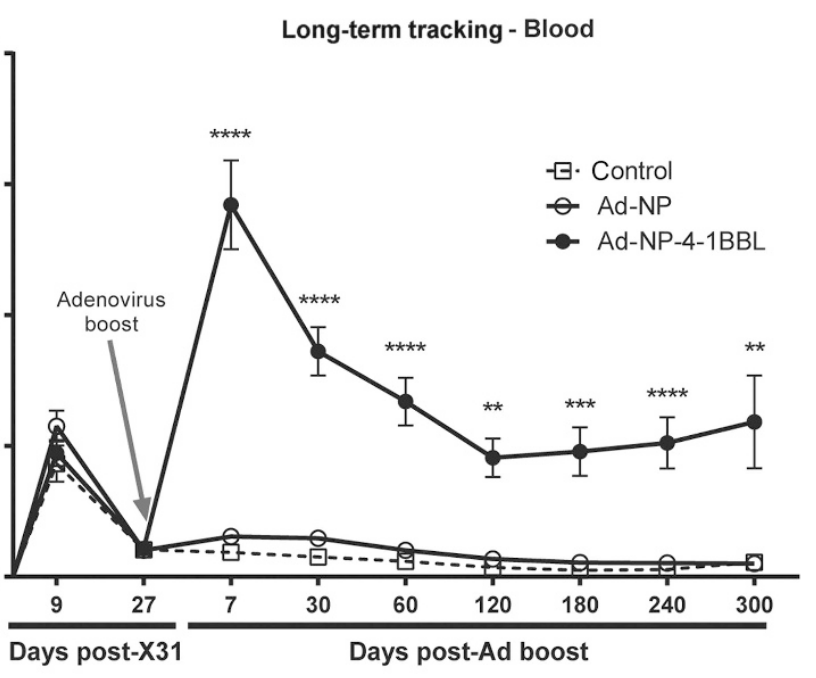

c

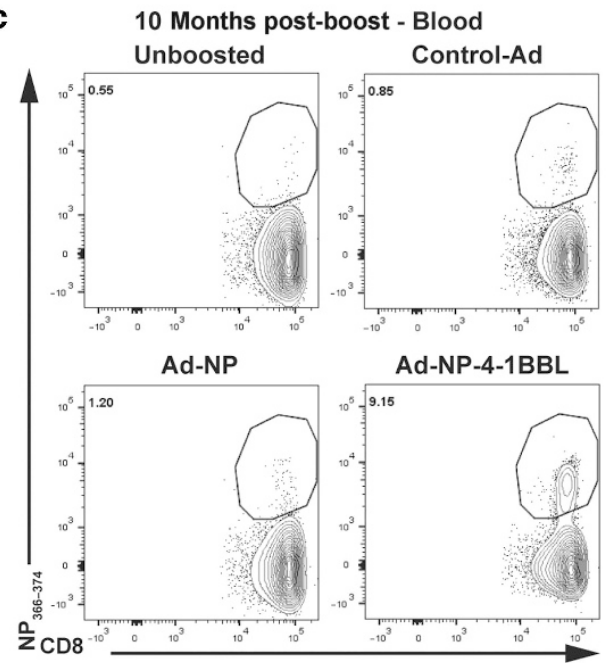

d

7 Months post-boost - Spleen
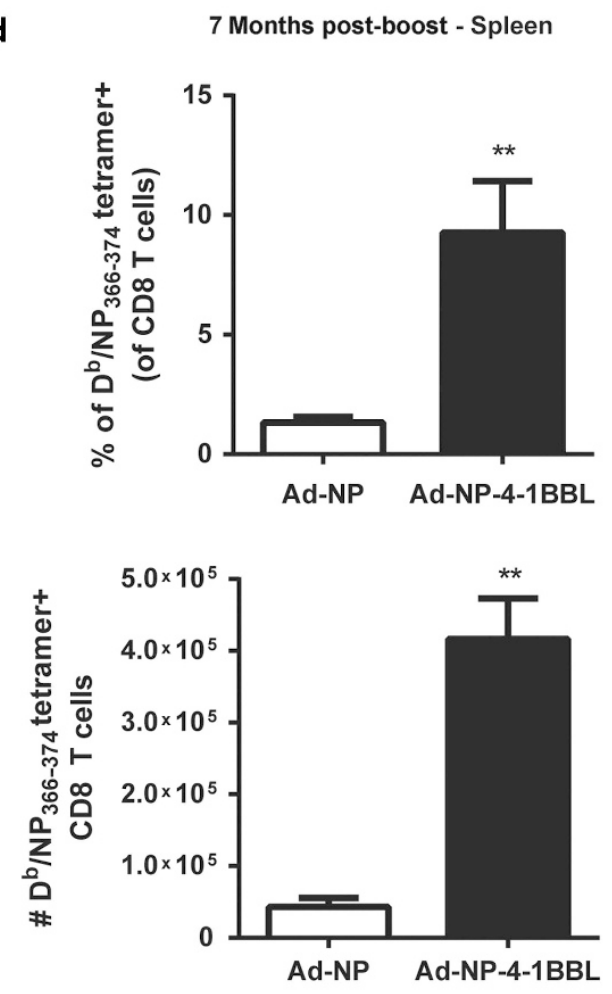

Figure 1 For caption see page 1295. e $\quad 9-11$ Months post-boost - PR8 Challenge
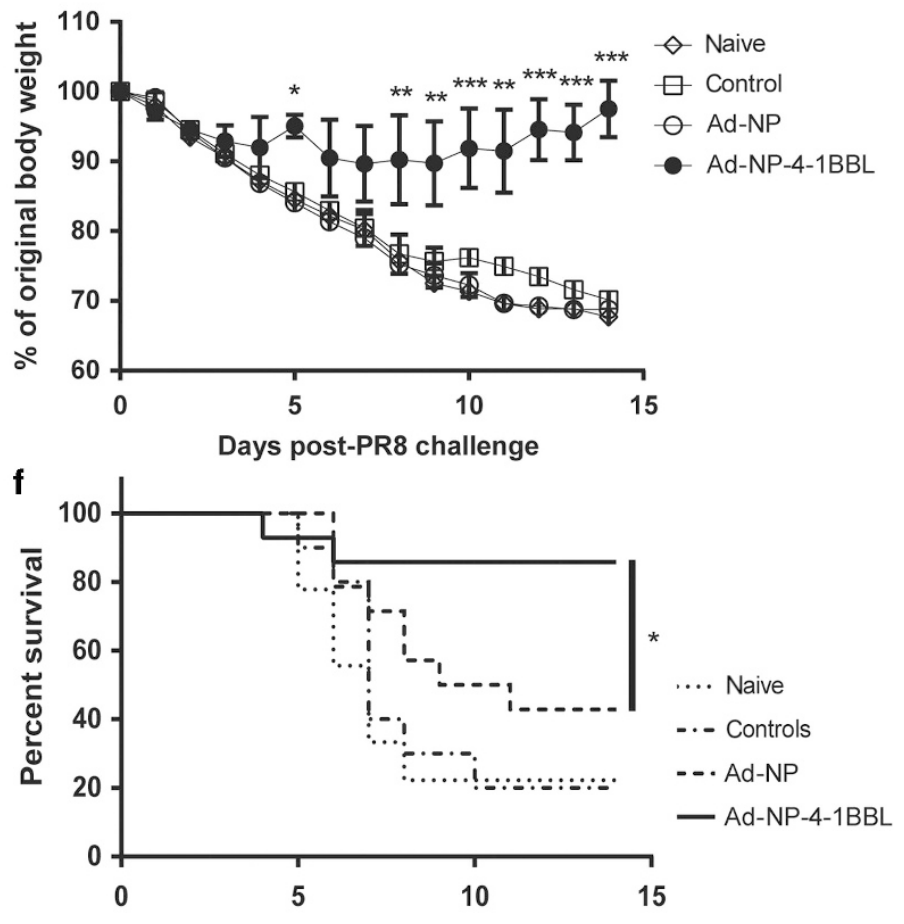

Days post-PR8 challenge 
a

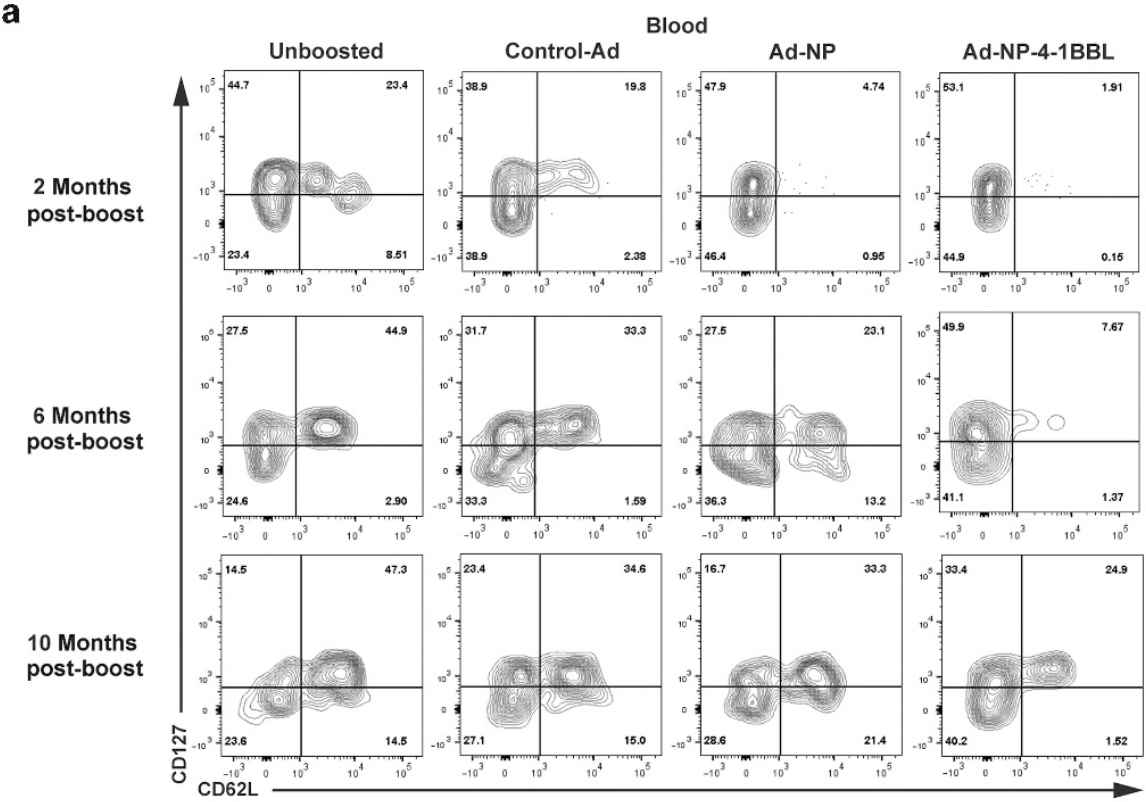

b

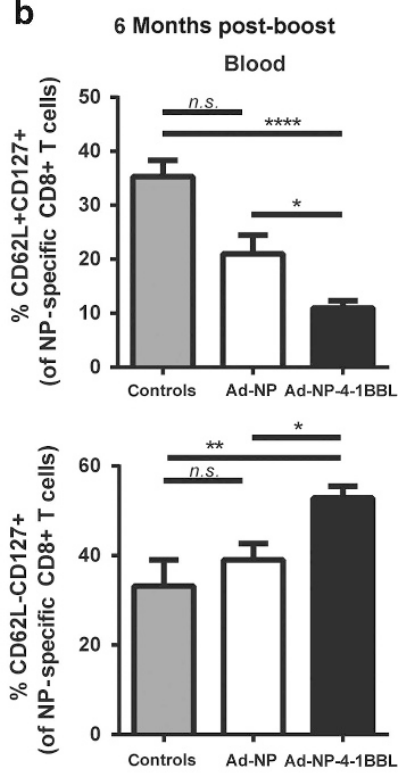

C

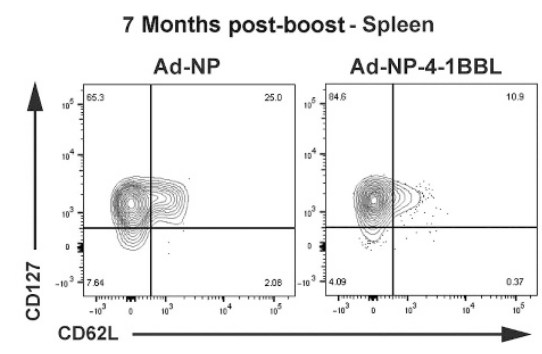

e

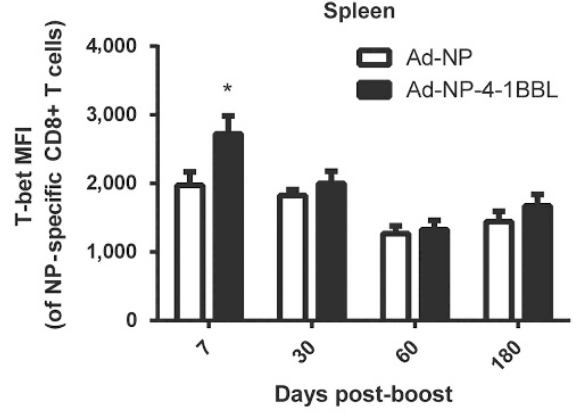

f

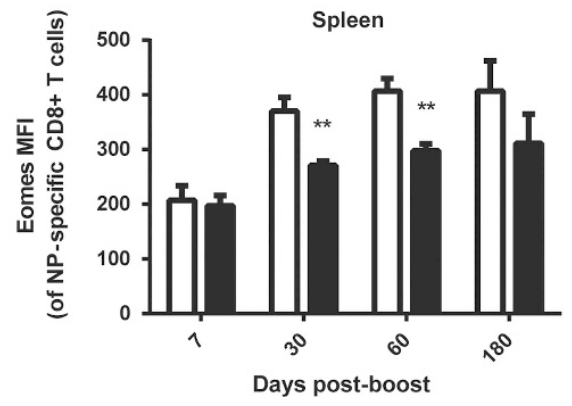

d 7 Months post-boost - Spleen
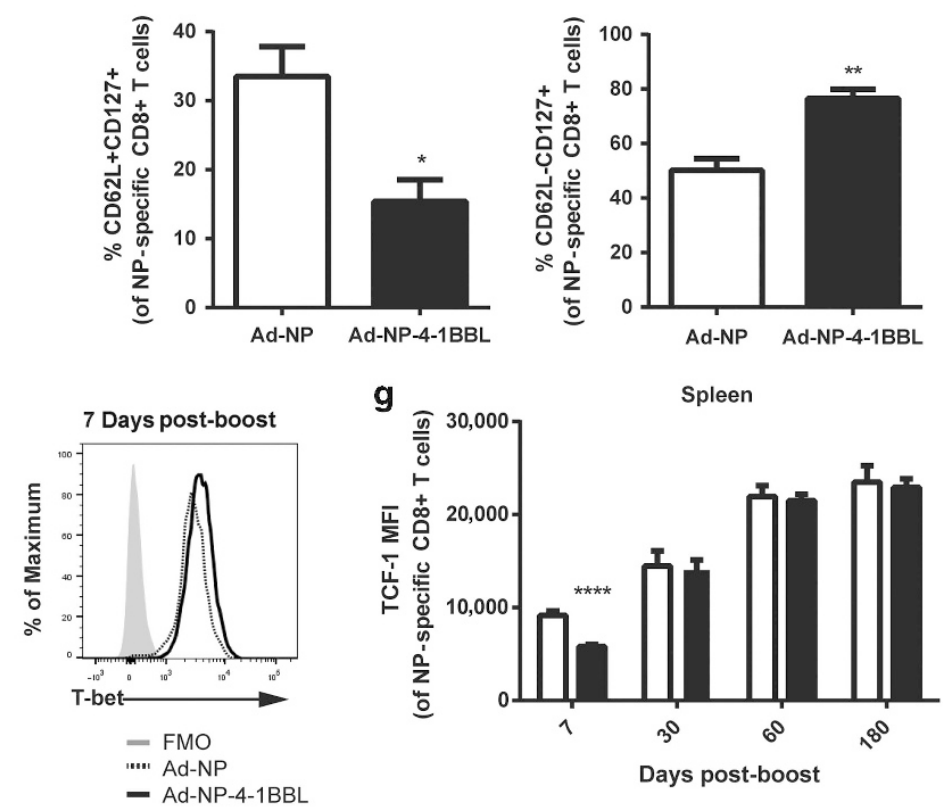

Spleen
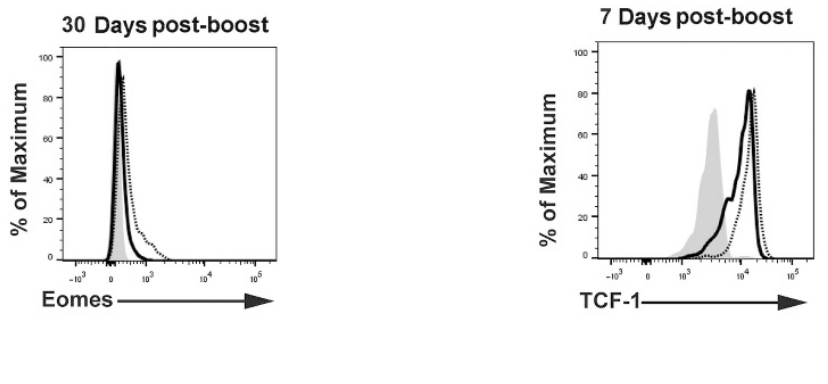

Figure 2 For caption see page 1298. 
reactivation. In contrast, Tem, which lack CD62L and CCR7, patrol the circulation and rapidly respond to antigen reencounter through IFN $\gamma$ and TNF $\alpha$ production. ${ }^{21,22} \mathrm{We}$ therefore evaluated the circulating memory $\mathrm{T}$ cells for CD62L as well as for CD127, the IL-7R $\alpha$ chain, to distinguish memory $\mathrm{T}$ cells from terminally differentiated effectors. ${ }^{23,24}$ $\mathrm{NP}_{366-374}$-specific CD8 T cells from Ad-NP-4-1BBL-boosted mice showed delayed acquisition of CD62L compared with Ad-NP or control Ad-boosted or unboosted mice (Figure 2a,b). This difference was first apparent at 2 months post-boost in the blood and persisted until at least 10 months. This decrease in $\mathrm{Tcm}$ and increase in Tem compartments was similarly observed in lymphoid organs, including the spleen (Figure 2c,d) and dLN (data not shown). The delayed acquisition of CD62L by the Ad-NP-4-1BBL-boosted $\mathrm{T}$ cells was not accompanied by lower CD127 expression (Figure 2a,c) and therefore not a result of expanded terminally differentiated effectors.

The T-box transcription factors T-bet and Eomesodermin (Eomes) are key drivers of CD8 T-cell lineage differentiation. The higher expression of T-bet or Eomes is associated with effector-like or memory-like phenotypes, respectively. ${ }^{25}$ Consistent with the effector-like nature of the memory CD8 T cells, the Ad-NP-4-1BBL boost induced higher T-bet expression early (day 7 post-boost, Figure 2e), and lower Eomes between day 30 and 60 (Figure 2f) compared with Ad-NP in the spleen. The transcription factor T-cell factor 1 (TCF-1) is induced downstream of the canonical Wnt pathway, and regulates memory CD8 T-cell differentiation and longevity, partially through the induction of Eomes. ${ }^{26}$ Splenic memory T cells from Ad-NP-4-1BBL-boosted mice have lower TCF-1 expression early post-boost (day 7, Figure $\mathbf{2 g}$ ). This effector-like transcriptional profile after 4-1BB stimulation appears to be a characteristic of secondary immunization, as $\mathrm{T}$ cells induced during primary immunization with Ad-NP or Ad-NP-4-1BBL were similar with respect to T-bet and Eomes profiles (data not shown). Differences in TCF-1 and T-bet were noted early (day 7) post-boost and thus are likely associated with memory programming rather than differences in the proportions of $\mathrm{Tcm}$ and Tem, which were not different at day 7 in the spleen when CD62L expression was very low in both Ad-NP and Ad-NP-41BBL-boosted groups (data not shown). By 6 months postboost, there is no longer a measureable difference in expression of these transcription factors between Ad-NP and Ad-NP-4-
1BBL boosted NP-specific CD8 T cells, whereas Tcm and Tem frequency differences persist (Figure $\mathbf{2 c}-\mathbf{g}$ ).

Consistent with the more effector-like memory phenotype, Ad-NP-4-1BBL boosting induced a significantly higher frequency, but not mean fluorescence intensity (MFI), of IFN $\gamma$ producing CD8 T cells, as determined by ex vivo restimulation with the $\mathrm{NP}_{366-374}$ peptide, even at 10 months post-boost when Ad-NP no longer differed substantially from controls (Figure 3a,b). Similar results were seen with TNF $\alpha$ and CD107a, with the latter showing a significantly higher MFI in Ad-NP-41BBL-boosted CD8 T cells. Ad-NP-4-1BBL-boosted mice also had more polyfunctional memory CD8 T cells, with a higher frequency of triple and quadruple producers of IFN $\gamma, \mathrm{TNF} \alpha$, CD107a, and IL-2 in the blood (Figure 3c), lung (Figure 3d), and spleen (Figure 3e). This polyfunctionality was not recapitulated with increasing doses of Ad-NP, which resulted in loss of functionality when titrated up 100-fold (Figure 3d,e), consistent with previously reported effects of high-dose Ad immunization. ${ }^{27}$ This loss of functionality at high Ad-NP dose occurs despite a greater number of NP tetramer ${ }^{+}$ $\mathrm{CD} 8 \mathrm{~T}$ cells in the lung and a fairly constant number in the spleen with increasing vector dose (Figure 3f). Thus, 4-1BBL in the boost phase induces a long-lived effector memory population that correlates with long-lived protection against influenza challenge.

\section{Local 4-1BB stimulation induces a long-lived lung-resident memory population consisting of both $\mathrm{CD}_{103}{ }^{+}$and CD103 $^{-}$subsets}

Trm are important mediators of protective immunity to influenza virus. ${ }^{8,10}$ Therefore, we investigated whether the local introduction of $4-1 \mathrm{BBL}$ with antigen through i.n. immunization impacted $\mathrm{T}$ cells in the lung. Intravascular infusion of labeled anti-CD8 antibody before euthanasia was used to distinguish vascular from parenchymal $\mathrm{T}$ cells that reside within the lung tissue (Figure 4a) ${ }^{28}$ Much like the circulating response characterized in the blood and spleen, there was a dramatic augmentation in the initial expansion of $\mathrm{NP}_{366-374^{-}}$ specific CD8 T cells in the lung tissue parenchyma that peaked at day 7 , and declined by day 60 to form a stable population that persisted to at least 7 months post-Ad-NP-4-1BBL boost (Figure 4a,b). This occurred at a dose where the response induced by Ad-NP alone diminished within the first 60 days. Whereas $\mathrm{NP}_{366-374}$-specific cells made up the majority of the

Figure 2 Ad-NP-4-1BBL i.n. boost induces an effector-like circulating memory CD8 T-cell population. (a) Tracking of central memory (Tcm; NP tetramer ${ }^{+} \mathrm{CD}_{127}{ }^{+} \mathrm{CD}_{2} \mathrm{~L}^{+}$) and effector memory (Tem; NP tetramer ${ }^{+} \mathrm{CD} 127^{+} \mathrm{CD}^{-} \mathrm{L}^{-}$) populations in the blood of mice left unboosted or boosted with control Ad, Ad-NP, or Ad-NP-4-1BBL, as described in Figure 1a. (b) Frequencies of Tcm and Tem were quantified at 6 months (day 180) post-boost in the blood. (c) Representative gating and (d) frequencies of Tcm and Tem at 7 months (day 210) post-boost in the spleen of Ad-NP and Ad-NP-4-1BBLboosted mice. Mean fluorescence intensity (MFI) and representative histograms of transcription factors (e) T-bet, (f) Eomesodermin (Eomes), and (g)

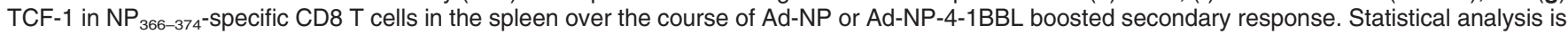
shown between Ad-NP and Ad-NP-4-1BBL boost within each time point. Data in $\mathbf{a}$ and $\mathbf{b}$ are pooled from two separate experiments with $n=6-8$ per group per experiment, whereas (c and d) are from two separate experiments with $n=3$ per group per experiment (mean \pm s.e.m.). (e-g) are pooled from two to three experiments per time point with $n=4$ per group per experiment (mean \pm s.e.m.). ${ }^{\star} P<0.05,{ }^{\star \star} P<0.005,{ }^{* * \star *} P<0.00005$ (Mann-Whitney test for b and $\mathbf{c}$ or Kruskal-Wallis test for $\mathbf{e}-\mathbf{g}$ ). 

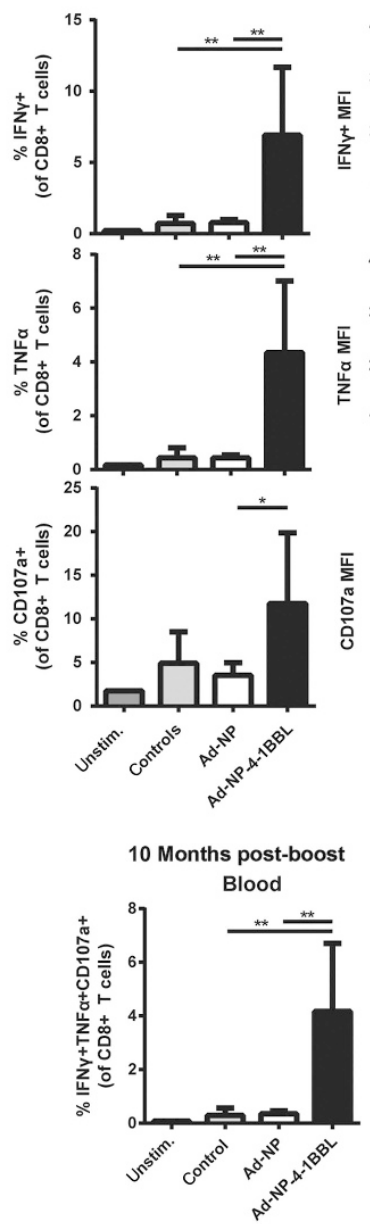
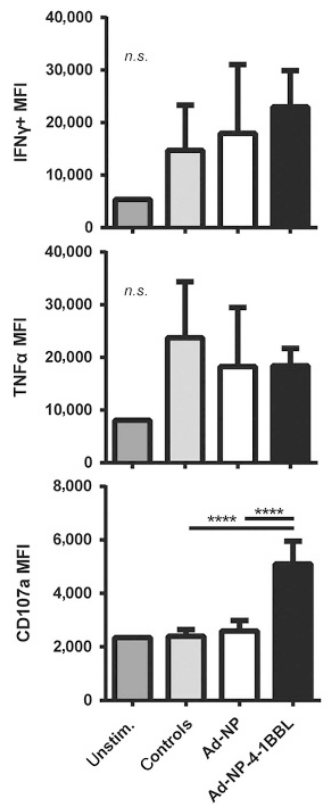

b
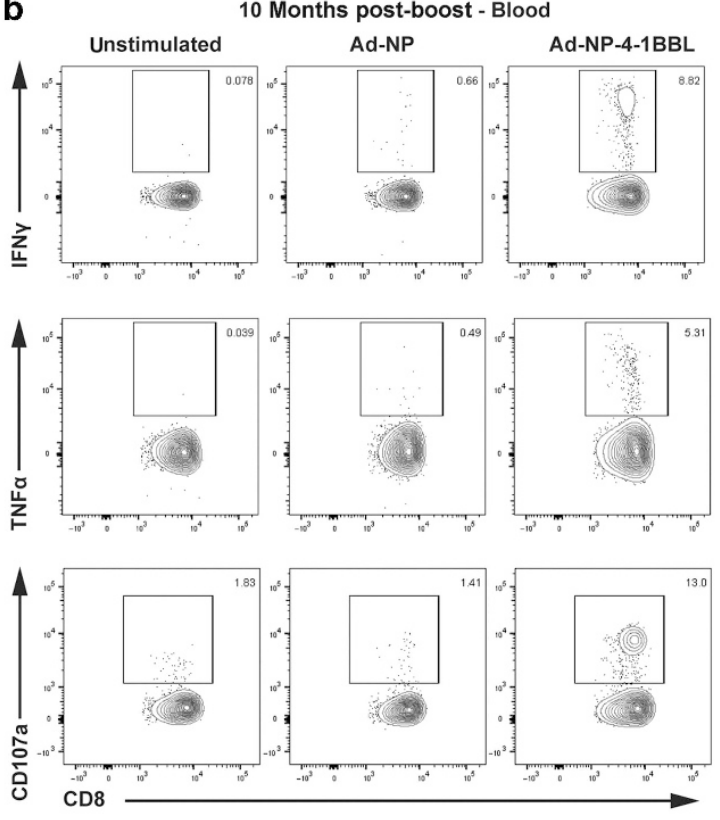

C

e

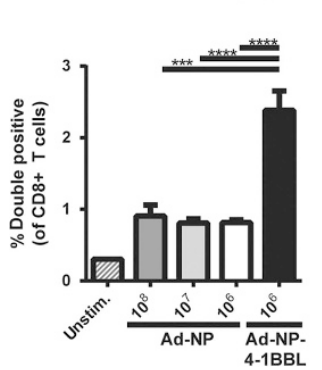

d 30 Days post-boost - Lung (IFNY, TNFa, CD107a, and IL-2)
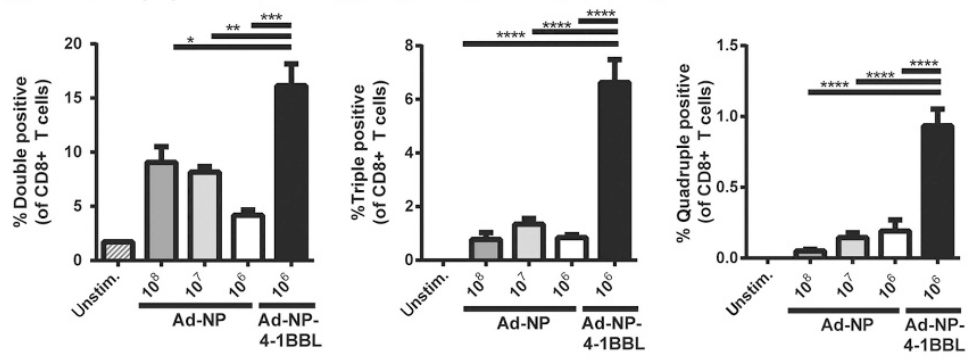

f 30 Days post-boost
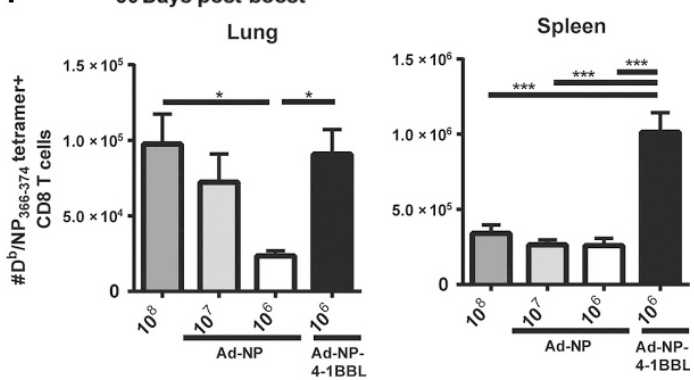

Figure 3 4-1BBL-boosted memory CD8 T cells are more polyfunctional at a lower dose of the adenovirus vector than NP-only boosted T cells. Samples were stimulated ex vivo with the $\mathrm{NP}_{366-374}$ peptide and intracellular staining was performed to assess (a) frequency and MFI of IFN $\gamma$, TNF $\alpha$, and CD107aproducing CD8 T cells in the blood at 10 months (day 300 ) post-boost with (b) representative gating. Frequency of polyfunctional CD8 T cells (c) in the blood at 10 months post-boost producing IFN $\gamma$, TNF $\alpha$, and CD107a; and at day 30 post-boost in the (d) lung and (e) spleen producing any two, three, or four combinations of IFN $\gamma$, TNF $\alpha$, CD107a, and IL-2 with Ad-NP dosage titrated up 10- and 100-fold. (f) Absolute numbers of $\mathrm{D}^{\mathrm{b}} / \mathrm{NP}_{366-374}$ tetramer ${ }^{+}$CD8 T cells in lung and spleen at 30 days post-boost with Ad-NP titration and Ad-NP-4-1BBL. Data in (a-c) are pooled from two separate experiments with $n=6-8$ per group per experiment, whereas (d-f) was performed once at day 30 and repeated independently with similar results at day 60 post-boost, all with $n=4$ mice per group per experiment (mean \pm s.e.m.). NS not significant, ${ }^{\star} P<0.05,{ }^{\star \star} P<0.005,{ }^{\star \star \star} P<0.0005,{ }^{\star \star \star \star} P<0.00005$ (Kruskal-Wallis test).

CD8 $\mathrm{T}$ cells within the lung tissue at their peak (day 7 postboost) in both Ad-NP and Ad-NP-4-1BBL-treated mice, the low numbers of $\mathrm{CD} 8 \mathrm{~T}$ cells that remained in the lung at 7 months after Ad-NP boost contained few that were $\mathrm{NP}_{366-374^{-}}$ specific (Figure 4c). This was in contrast to Ad-NP-4-1BBLboosted mice, where the stable population of Trm was largely $\mathrm{NP}_{366-374}$ specific. These persistent lung parenchymal NP- specific $\mathrm{T}$ cells from the Ad-NP-4-1BBL-boosted mice consisted of $\mathrm{CD}_{103}{ }^{+} \mathrm{CD} 69^{+}, \mathrm{CD} 103^{-} \mathrm{CD} 69^{+}$, and $\mathrm{CD}_{103}{ }^{-} \mathrm{CD} 9^{-}$cells (Figure 4a,d). $\mathrm{CD} 103{ }^{+} \mathrm{CD} 69^{+}$Trm have previously been identified in the lung, ${ }^{29}$ whereas $\mathrm{CD} 103^{-}$ $\mathrm{CD}^{2} 9^{+} \mathrm{Trm}$ have been reported in the lamina propria, secondary lymphoid organs, and thymus. ${ }^{7}$ Both these populations expressed low T-bet, Eomes, and CD127, as well as high 

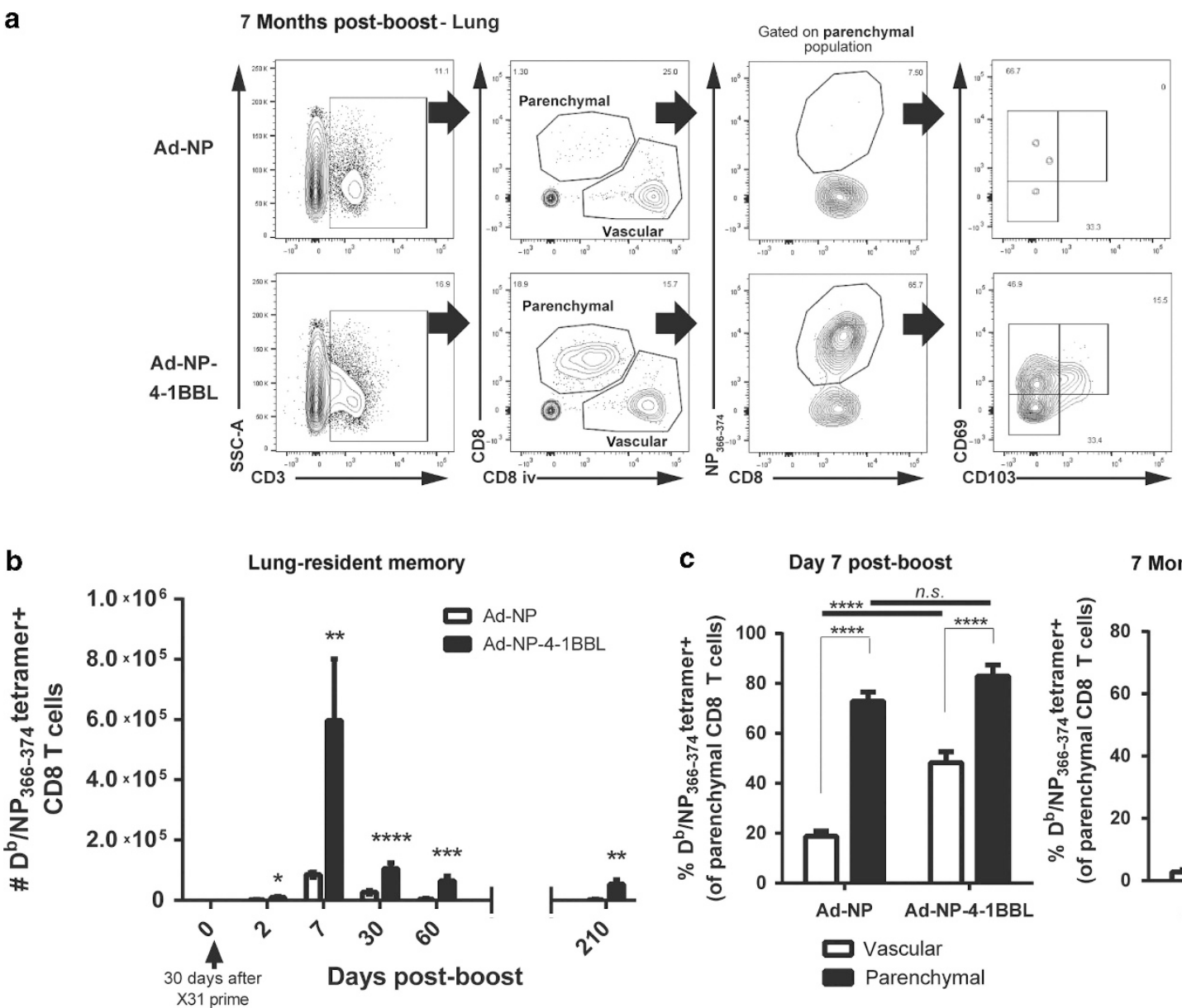

d

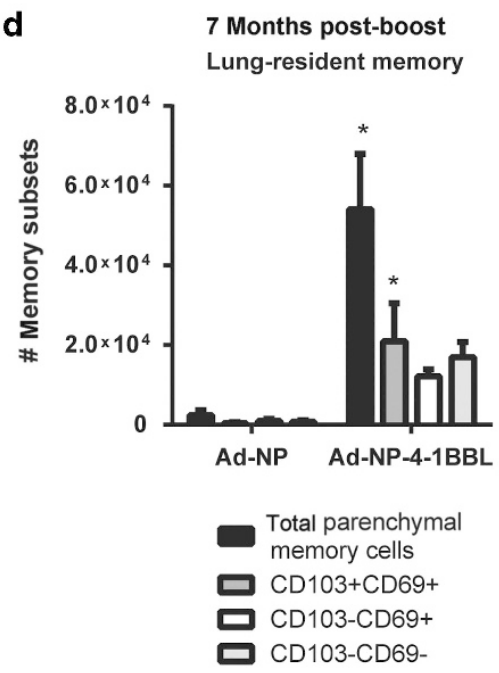

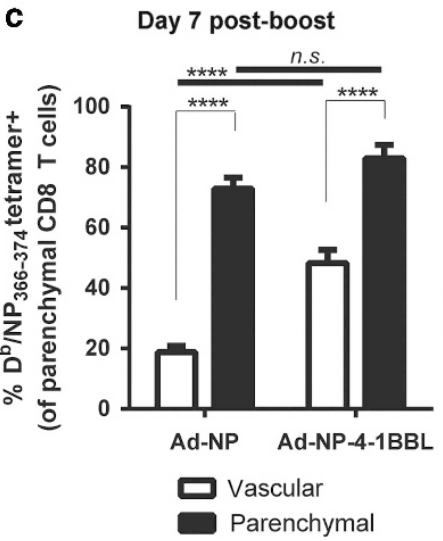

7 Months post-boost

$-4-1 B B L$

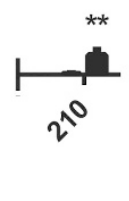

e

7 Months post-boost - Lung (Ad-NP-4-1BBL boost)
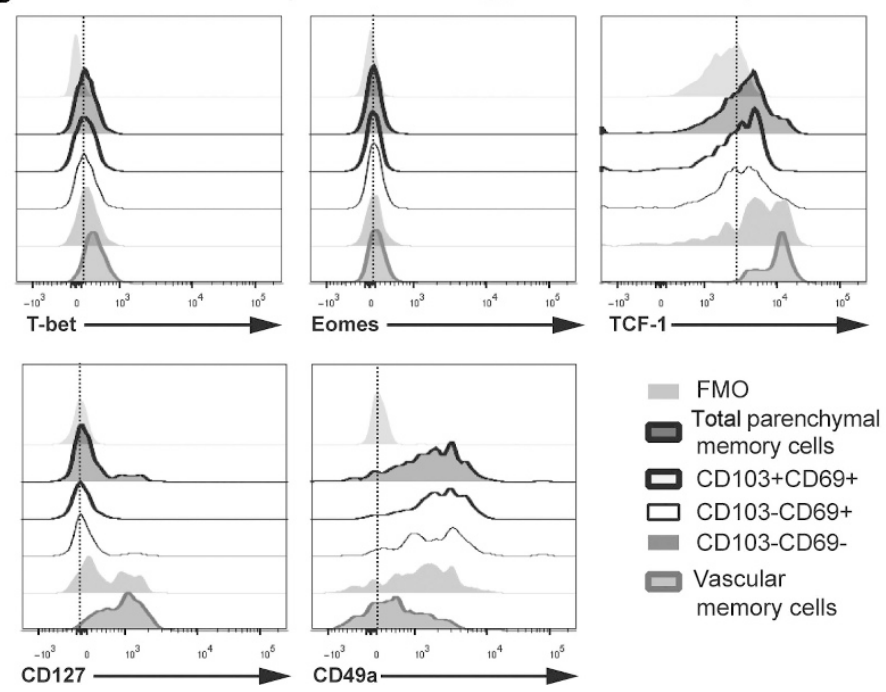

- FMO

Total parenchymal

memory cells

CD103+CD69+

$\square$ CD103-CD69+

- CD103-CD69-

Vascular

memory cells

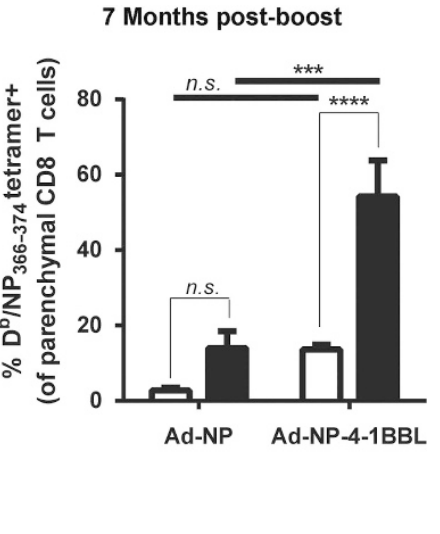

Figure 4 Ad-NP-4-1BBL i.n. boost induces a persistent population of $\mathrm{CD}_{103^{+}}$and $\mathrm{CD}_{103^{-}}$lung-resident populations of memory CD8 $\mathrm{T}$ cells. (a) Gating strategy for intravascular antibody staining to differentiate vascular and parenchymal lung populations at 7 months (day 210 ) post-boost in the

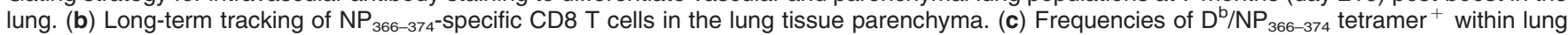
parenchymal CD8 T cells at day 7 and 7 months post-Ad-NP or Ad-NP-4-1BBL boost. (d) Absolute numbers of total NP $366-374^{-s_{3}}$ ecific memory CD8 T cells, and subpopulations of $\mathrm{CD}_{103}{ }^{+} \mathrm{CD} 9^{+}, \mathrm{CD}_{103}{ }^{-} \mathrm{CD} 69^{+}$, and $\mathrm{CD} 103^{-} \mathrm{CD}_{6} 9^{-}$memory $\mathrm{CD} 8 \mathrm{~T}$ cells in the lung tissue at 7 months post-boost. (e) Flow cytometry analysis of T-bet, Eomes, TCF-1, CD127, and CD49a MFI between total lung parenchymal, parenchymal CD $103^{+}$CD69 ${ }^{+}$, parenchymal $\mathrm{CD}_{103^{-}} \mathrm{CD} 9^{+}$, parenchymal CD103 ${ }^{-} \mathrm{CD}^{-} 9^{-}$, and lung vascular memory CD8 T cells. Data were pooled from two separate experiments per time point with $n=3$ per group per experiment (mean \pm s.e.m.). NS not significant, ${ }^{\star} P<0.05,{ }^{\star \star} P<0.005,{ }^{\star \star \star} P<0.0005,{ }^{\star \star * \star} P<0.00005$ (two-way ANOVA test). 
a

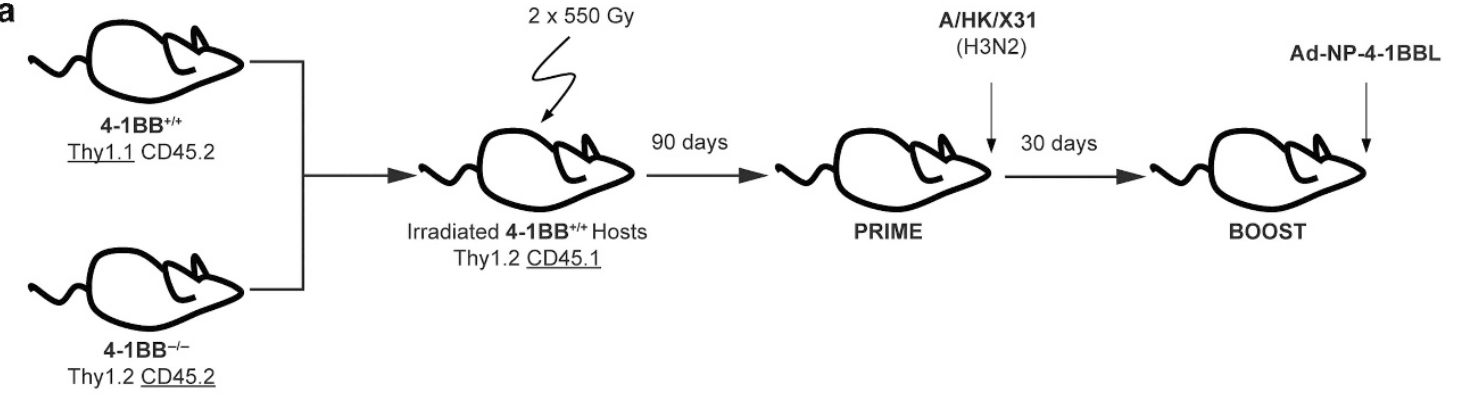

b

Pre-infection - blood (CD45.2+ donors; Thy1.1+:Thy1.1-)

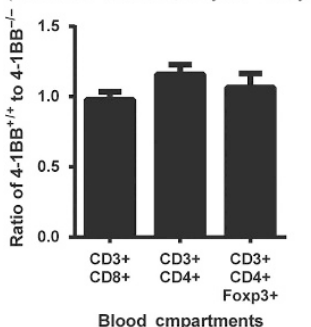

C

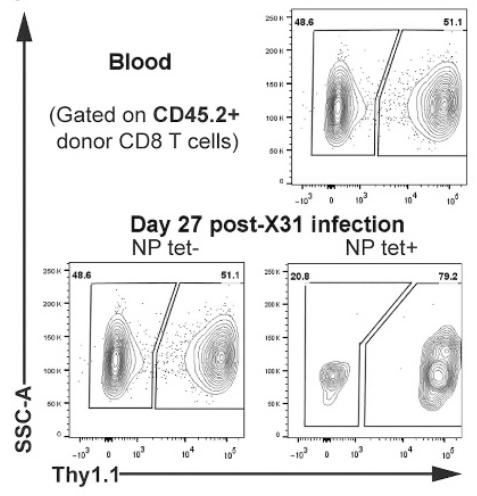

d

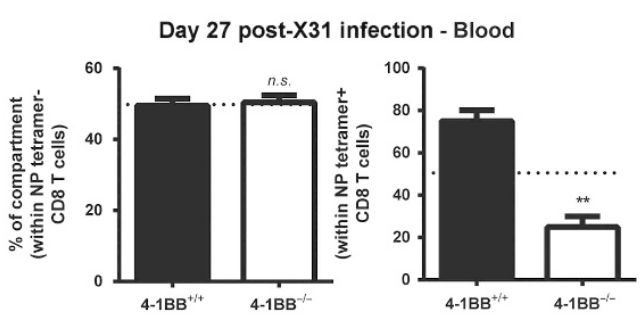

e Day 7 post-boost

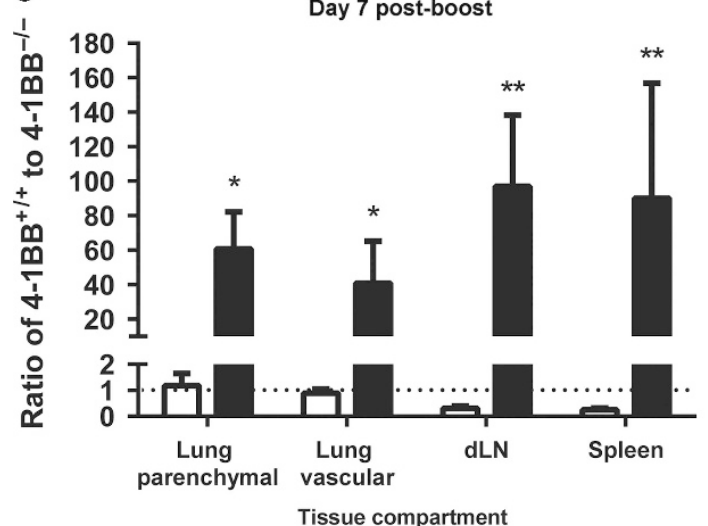

f

Day 7 post-boost (CD45.2+ NP tetramer+ CD8 T cells) Lung
Lung
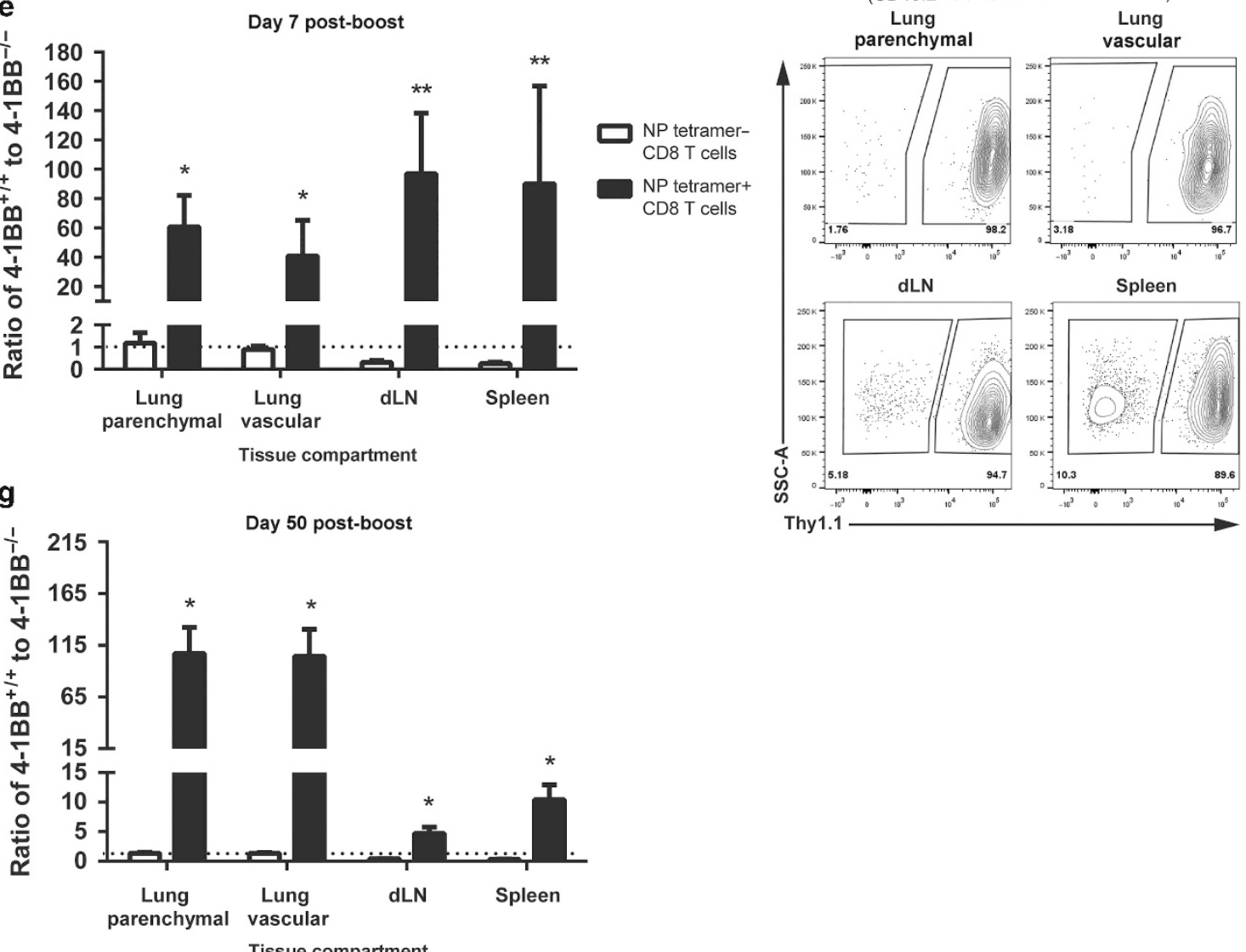

NP tetramerCD8 T cells

NP tetramer+ CD8 T cells

Tissue compartment

Figure 5 For caption see page 1302. 
lung-homing CD49a compared with lung vascular memory populations (Figure 4e), as previously reported for lung Trm. ${ }^{28,30-33}$ We also observed lower TCF-1 expression in the lung NP-specific Trm. The $\mathrm{CD}_{103}{ }^{-} \mathrm{CD}_{69^{-}}$population had an intermediate phenotype between the vascular and the $\mathrm{CD} 69^{+}$ parenchymal populations for many of these markers. Thus, stimulating $4-1 \mathrm{BB}$ directly in the respiratory tract induces a robust enlargement of the Trm population over control Ad-NP immunization, and this effect persists to at least 210 days postboost.

\section{Ad-NP-4-1BBL acts directly on the CD8 $T$ cells to induce expansion in local and systemic tissue compartments}

To determine if $4-1 \mathrm{BB}$ was acting directly on the $\mathrm{CD} 8 \mathrm{~T}$ cells or through another cell type, we used mixed bone marrow (BM) chimeras in which lethally irradiated mice were reconstituted with a $1: 1 \mathrm{mix}$ of $4-1 \mathrm{BB}^{+/+}$and $4-1 \mathrm{BB}^{-1-} \mathrm{BM}$. Blood was monitored for degree of chimerism at 3 months postreconstitution, followed by priming with influenza A/X31. One month later, mice were given Ad-NP-4-1BBL by the i.n. route (Figure 5a). Before infection, the ratio of $4-1 \mathrm{BB}^{+/+}$to 4 $1 \mathrm{BB}^{-1-} \mathrm{CD} 8 \mathrm{~T}$ cells was $\sim 1: 1$ (Figure $5 \mathbf{b}, \mathbf{c}$ ). At 27 days after infection with X31, there was already a 3- to 4 -fold advantage of $4-1 \mathrm{BB}^{+/+}$over $4-1 \mathrm{BB}^{-1-} \mathrm{NP}_{366-374}$-specific CD8 $\mathrm{T}$ cells in the blood, whereas the non-NP-specific CD8 T-cell compartment remained at the 1:1 ratio of the initial reconstitution (Figure $\mathbf{5 c}$,d). These data recapitulate previous studies on the role of $4-1 \mathrm{BB} / 4-1 \mathrm{BBL}$ in maintaining the circulating CD8 T-cell memory pool. ${ }^{14}$ At day 7 post-Ad-NP-4-1BBL boost (the peak response), the $\mathrm{NP}_{366-374}$-specific CD8 $\mathrm{T}$ cells were almost exclusively from the $4-1 \mathrm{BB}^{+/+}$donor in the lung parenchyma, lung vasculature, $\mathrm{dLN}$, and spleen (Figure 5e,f), and this dichotomy persisted out to day 50 post-boost (Figure 5g). Thus the effects of 4-1BBL on the circulating and the lung-resident NP-specific memory CD8 T-cell populations are completely dependent on direct (intrinsic) effects of 4-1BB on the CD8 T cells.

\section{Ad-NP-4-1BBL induces greater IL-7R $\alpha$ (CD127) expression on memory CD8 T cells, which may contribute to the longevity of the response}

We next asked if 4-1BB stimulation leads to long-lived memory $\mathrm{T}$ cells through effects on cytokine receptors associated with T-cell survival, memory commitment, and maintenance, including CD25 (IL-2R $\alpha$ ) and CD122 (IL-2R $\beta$ ) and CD127 (the IL-7R $\alpha$ subunit). CD25 and CD122, although present, did not show consistent differences between the Ad-NP and AdNP-4-1BBL groups (data not shown). However, Ad-NP-41BBL-boosted mice exhibited a higher frequency of $\mathrm{CD} 127^{+}$ $\mathrm{NP}_{366-374}$-specific memory CD8 $\mathrm{T}$ cells in the lung parenchyma but not lung vasculature compared with Ad-NP-boosted mice as early as day 7 post-boost, with similar results in the dLN. Modest frequency differences persisted in the spleen at day 30 (Figure 6a,b), but were no longer apparent by day 60 in any of the tissues examined (data not shown). Of note, the NP-specific CD8 T cells from the 4-1BBL-boosted group expressed higher CD127 at the per cell level from day 30 (Figure 6c) to at least 6 months post-boost (Figure 6d,e). MFI of CD127 was examined on total lung tissue at this time point instead of separated into lung parenchymal and vascular compartments because there were very few memory CD8 T cells left in the lung tissue in the Ad-NP group late in the boost response. The higher frequency and per cell expression of CD127 was specific to $4-1 \mathrm{BB}$ stimulation in the secondary response as mice given Ad-NP or Ad-NP-4-1BBL i.n. as a primary immunization did not exhibit this difference (data not shown).

To test the importance of IL7R $\alpha$ induction in the boost phase, we used an anti-CD127 blocking antibody (Figure 6f). IL-7R blockade at days 0 and 2 post-boost decreased the number of NP-specific memory $\mathrm{T}$ cells in the spleen by a similar proportion, regardless of whether the boost contained Ad-NP or Ad-NP-4-1BBL (Figure 6g), consistent with a critical role for IL-7R $\alpha$ in general. However, the lung Trm population was not affected by IL-7R blockade (data not shown). Whether this is because the systemic antibody does not effectively penetrate the lung parenchyma to block IL-7R or whether IL-7R is dispensable for the Trm cannot be distinguished in this experiment. Thus, $4-1 \mathrm{BBL}$ in the boost phase induces a sustained elevation of IL-7R $\alpha$ levels on the NP-specific CD8 memory $\mathrm{T}$ cells, which may contribute to their increased persistence; however, it is difficult to ascertain the significance of this increase as IL-7R is critical for the memory response regardless of inclusion of 4-1BBL in the boost.

\section{Delivery of 4-1BBL directly into the airways is required for the lung-resident memory response}

We next asked whether existing CD8 Trm populations in the lung can be boosted by a systemic administration of 4-1BBL. Primary infection with low-dose X31 i.n. induced a small

Figure 5 Ad-NP-4-1BBL acts directly on the CD8 T-cell compartment to induce enhanced localized and circulating memory responses. (a) Mixed bone marrow (BM) chimeras were generated by reconstituting a 1:1 ratio of 4-1BB ${ }^{+1+}$ (Thy1.1 CD45.2) and 4-1BB ${ }^{-1-}$ (Thy1.2 CD45.2) BM into lethally irradiated 4-1BB ${ }^{+/+}$(Thy1.2 CD45.1) host mice. Chimeric mice were rested for 90 days and then infected i.n. with A/HK/X31 infection followed by Ad-NP4-1BBL 1 month later. (b) Ratios of 4-1BB ${ }^{+1+}$ to $4-1 \mathrm{BB}^{-1-}$ of CD8 T cells, CD4 T cells, and Treg compartments in the blood of chimeric mice after reconstitution and before immunization. (c) Representative flow plot for donor CD8 T cells in blood pre- and 27 days post-infection. (d) Ratios of CD45.2 donor 4-1BB $+1+$ to $4-1 \mathrm{BB}^{-1-}$ of $\mathrm{NP}_{366-374}$ tetramer-negative and $\mathrm{NP}_{366-374}$ tetramer-positive CD8 T cells in the blood at 27 days post-X31 infection, with dotted line indicating pre-infection reconstitution ratios in the blood. $(\mathbf{e}-\mathbf{g})$ Ratios of CD45.2 donor 4-1 $\mathrm{BB}^{+/+}$to 4-1BB ${ }^{-1-}$ of $\mathrm{NP}_{366-374}$ tetramer-negative and $\mathrm{NP}_{366-374}$ tetramer-positive CD8 T cells in the lung parenchyma, lung vasculature, dLN, and spleen at (e) 7 days with (f) representative flow plots, and (g) day 50 post-Ad-NP-4-1BBL-boost. Statistical analysis is shown between tetramer positive and negative CD8 T cells within each organ. Data from (b, d, and e) pooled from two separate experiments with $n=8$ per experiment, whereas $(\mathbf{g})$ is pooled from two experiments with $n=3$ per experiment (mean \pm s.e.m.). ${ }^{\star} P<0.05,{ }^{\star *} P<0.005$ (Wilcoxon test). 
a

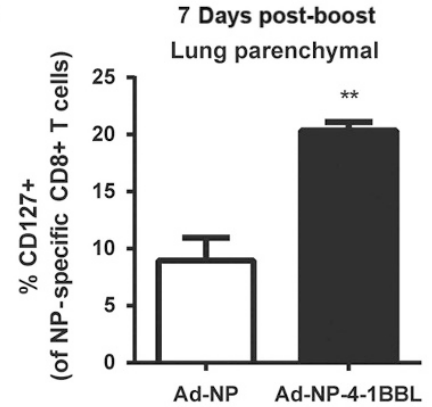

b
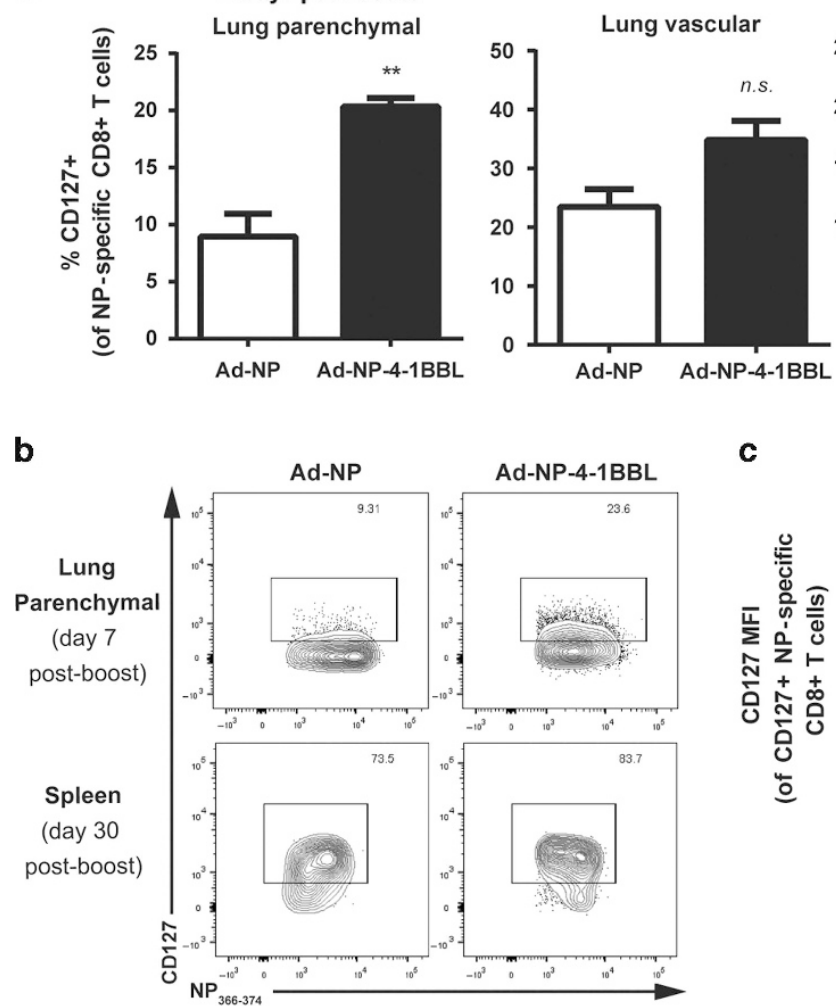

c

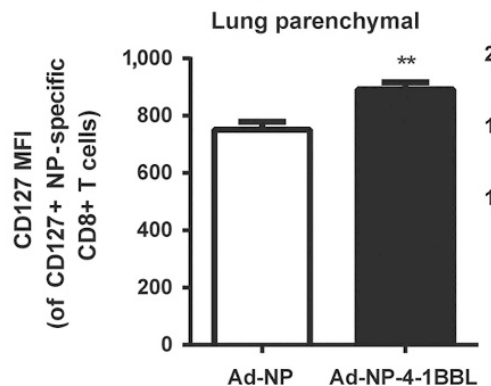

30 days post-boost Spleen

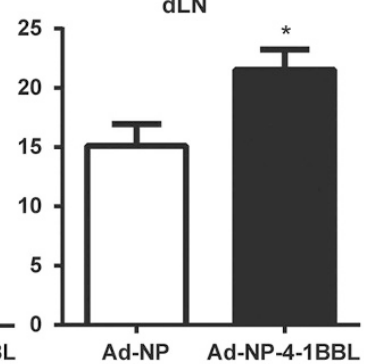

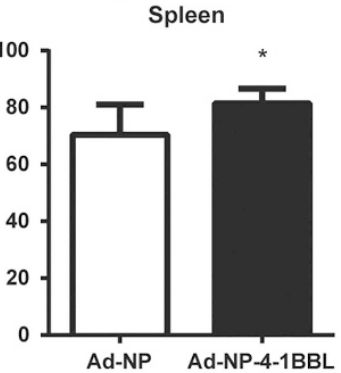

e

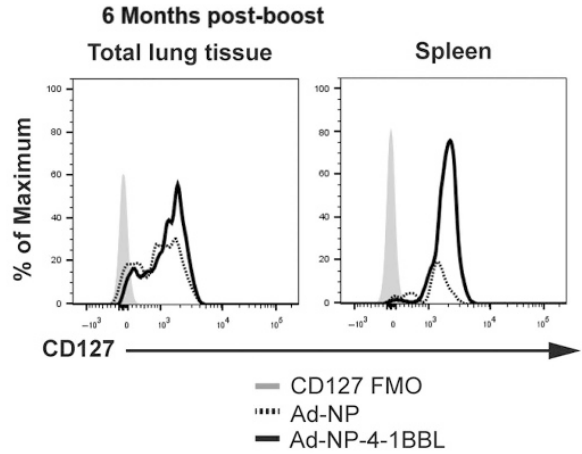

d

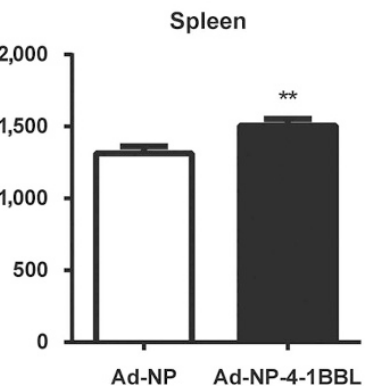

6 Months post-boost

Total lung tissue
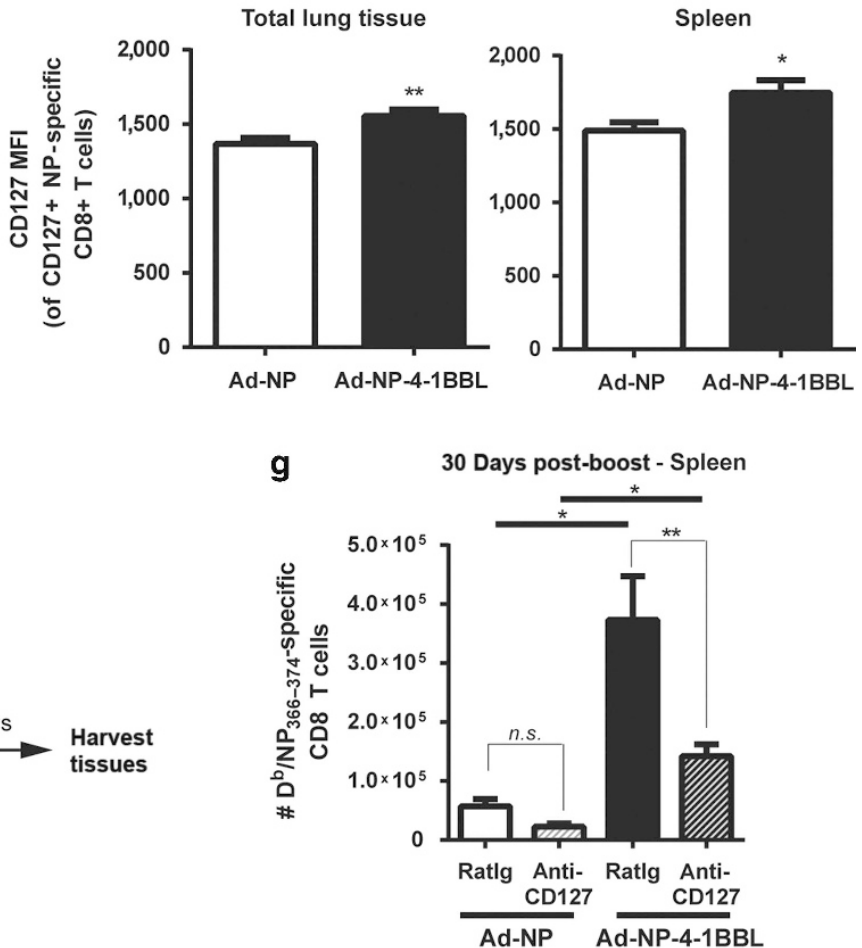

Ad-NP or Ad-NP-4-1BBL

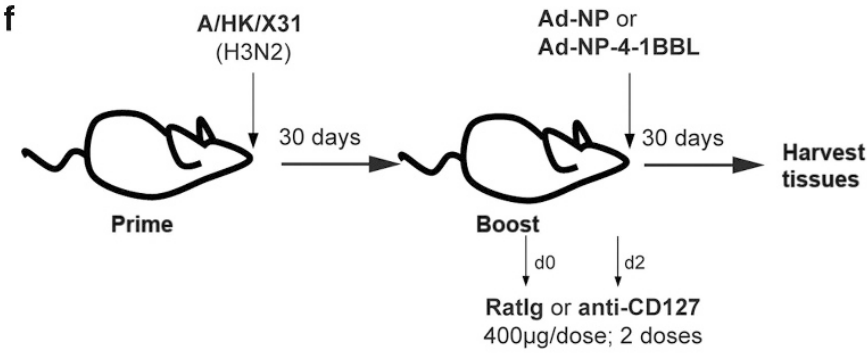

Figure 6 Ad-NP-4-1BBL-boosted memory cells have increased CD127 (IL-7R $\alpha$ ) expression. (a) Frequency of CD127 expression on NP $366-374$-specific memory CD8 T cells in the lung parenchyma, lung vasculature, and dLN at day 7; and the spleen at day 30 post-Ad-NP or Ad-NP-4-1BBL-boost, with (b) representative gating of the lung tissue and spleen. MFI of CD127 in the lung and spleen at (c) day 30 and (d) 6 months (day 180) post-boost, with (e) representative histograms. (f) Mice were infected i.n. with X31 and then boosted 30 days later with either Ad-NP or Ad-NP-4-1BBL along with an i.p. injection of Ratlg or anti-CD127 (A7R34) blocking antibody at day 0 and day 2 post-boost ( $400 \mu \mathrm{g}$ per dose). (g) NP $366-374$-specific CD8 T cells were enumerated in the spleen at day 30 post-boost. Data are pooled from two (a (day 7), d) or three (a (day 30)-c separate experiments with $n=4$ per group per experiment (mean \pm s.e.m.). NS not significant, ${ }^{*} P<0.05,{ }^{* \star} P<0.005$, ${ }^{* \star \star} P<0.0005$ (Mann-Whitney test). 
a
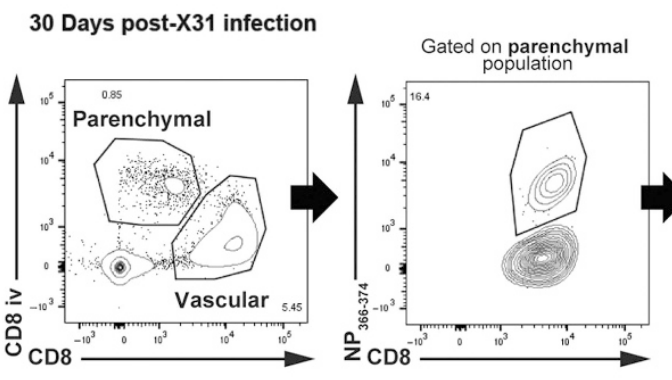

c
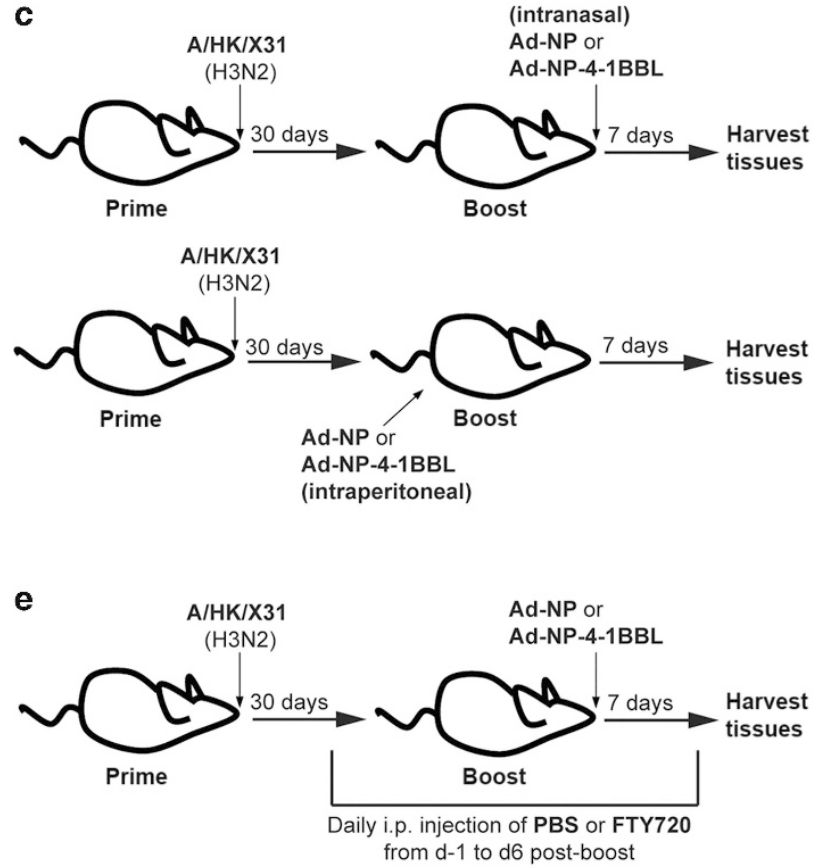

g
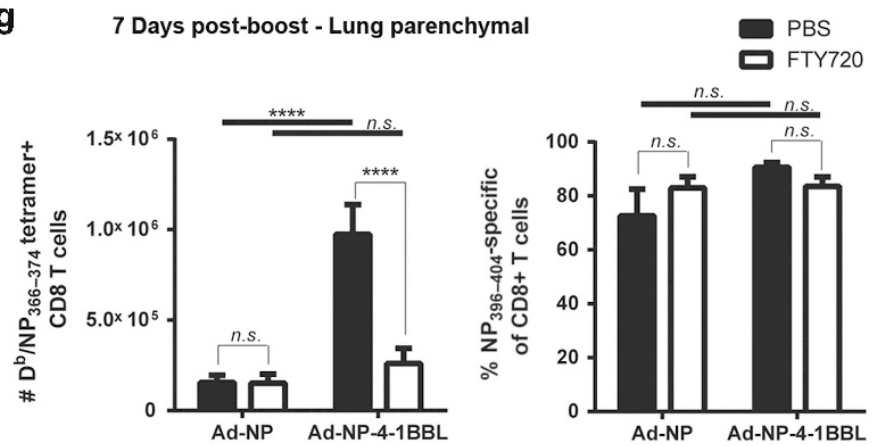

d
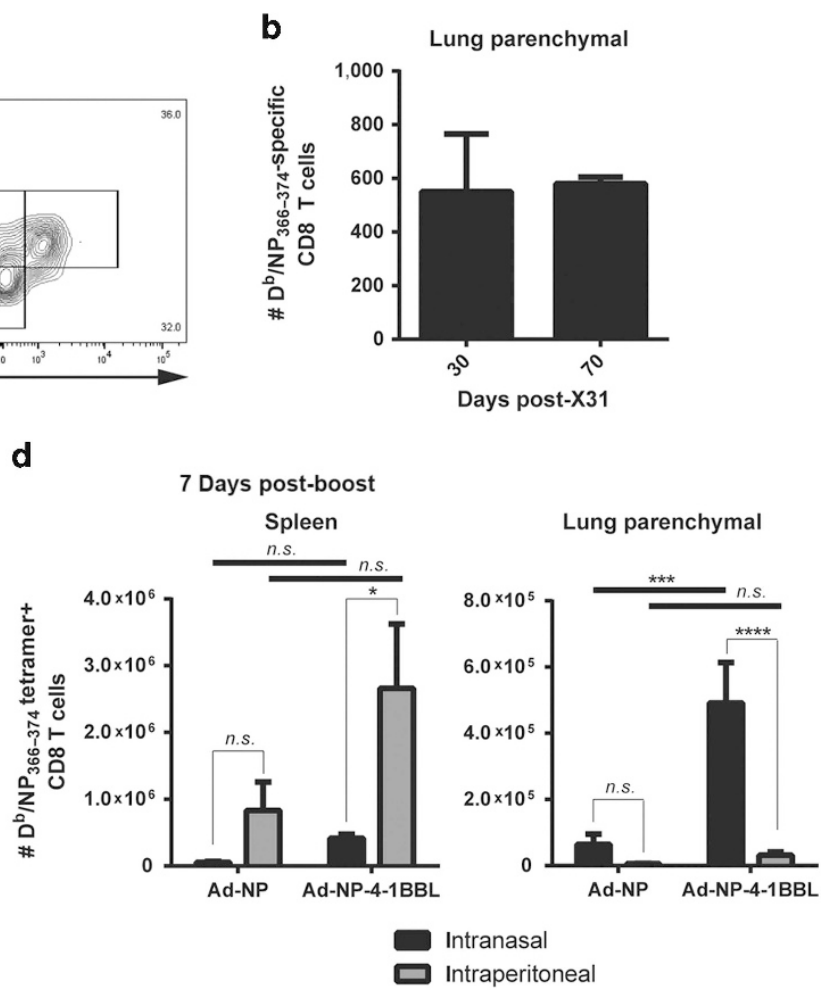

f

7 Days post-boost - Blood

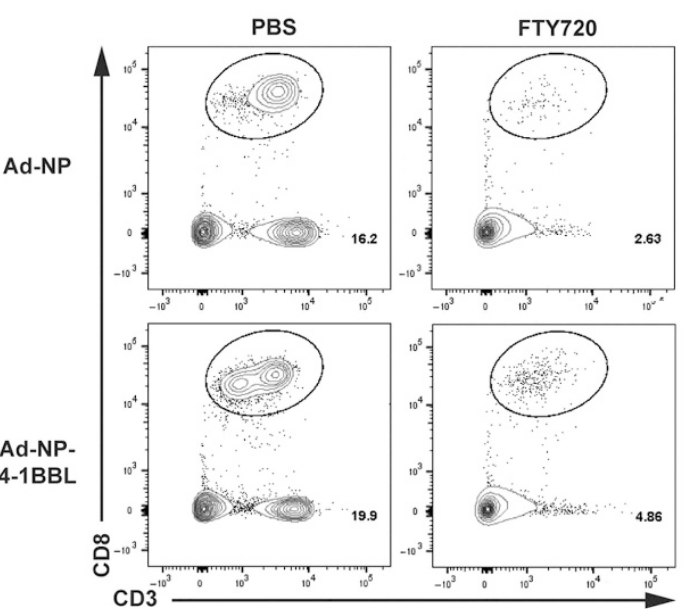

Figure 7 Delivery of 4-1BB stimulation directly into the airways by i.n. immunization is required for lung parenchymal secondary memory expansion, which relies predominantly on recruitment of antigen-specific CD8 T cells from the circulation. (a) Representative gating and (b) absolute number of lung parenchymal memory CD8 T cells at 30 and 70 days after i.n. X31 infection. In order to assess whether systemic 4-1BBL-boost can expand local Trm, (c) mice were infected i.n. with A/HK/X31 (5 HAU/mouse) and 1 month later boosted with Ad-NP or Ad-NP-4-1BBL either by i.n. or i.p. immunization (106 ${ }^{6}$ pfu per mouse). (d) Expansion of $\mathrm{NP}_{366-374}$-specific memory CD8 T cells at day 7 post-boost peak response is quantified in the lung and spleen. (e) Mice were infected with Influenza A/X31 and then 1 month later boosted i.n. with either Ad-NP or Ad-NP-4-1BBL and given an i.p. injection of either PBS control or FTY720 (1 mg kg $\left.{ }^{-1}\right)$ daily, until killed at day 7 post-boost. (f) Representative flow plots confirm sequestration of CD8 T cells from the circulation. (g)

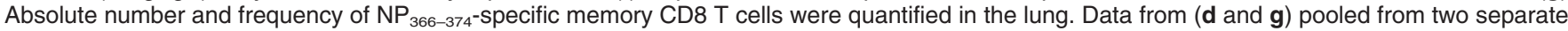
experiments with $n=4$ per group per experiment (mean \pm s.e.m.). For a, day 30 was pooled from two experiments while day 70 is only one experiment, all $n=4$ per experiment. NS not significant, ${ }^{\star} P<0.05$, ${ }^{\star \star \star} P<0.0005$, ${ }^{\star * \star \star} P<0.00005$ (two-way ANOVA test).

number of lung parenchymal $\mathrm{NP}_{366-374}$-specific memory CD8 $\mathrm{T}$ cells, which remained stable between days 30 and 70 postinfection in the tissue (Figure 7a,b). Mice were boosted at 30 days after the i.n. prime with Ad-NP or Ad-NP-4-1BBL by either the i.n. or the i.p. route (Figure 7c). As expected, by day 7 the systemic i.p. boost had resulted in a much greater peak $\mathrm{NP}_{366-374}$-specific memory CD8 T-cell response in the spleen compared with i.n. administration (Figure 7d). However, i.p. 
boost did not result in efficient accumulation of memory CD8 $\mathrm{T}$ cells in the lung, despite i.n. priming immunization. Importantly, systemic delivery of Ad-NP-4-1BBL did not significantly increase the number of $\mathrm{NP}_{366-374}$-specific $\mathrm{CD} 8 \mathrm{~T}$ cells in the lung compared with Ad-NP alone. Thus, local delivery of the 4-1BBL-containing vector is required for lung-resident Trm accumulation in response to $4-1 \mathrm{BBL}$ during the boost phase.

\section{Expansion of Trm during boosting is dependent on recruitment of $T$ cells into the lung}

The increase in Trm following intranasal boost with Ad-NP-4$1 \mathrm{BBL}$ could be due to expansion of existing memory $\mathrm{T}$ cells in the lung or recruitment of new effector T cells into the Trm pool. To distinguish these possibilities, we treated influenzaprimed mice daily by i.p. injection of either PBS or the immunomodulatory drug FTY720 during the boost phase (Figure 7e). FTY720 causes internalization of the sphingosine 1-phosphate receptor resulting in the sequestration of lymphocytes within lymph nodes, thereby preventing lymphocytes from entering the circulation. ${ }^{34}$ Thus, any CD8 memory T-cell expansion within the lung would be a result of 4-1BBL acting on populations already present in the lung before adenoviral boost. As expected, FTY720 treatment caused an increase of $\mathrm{NP}_{366-374}$-specific memory CD8 T cells within the dLN (data not shown), and a decrease in the blood (Figure 7f) and spleen (data not shown). Without $\mathrm{T}$ cells trafficking into the lungs, there was a significant reduction of NP-specific cells in the tissue parenchyma after Ad-NP-4-1BBL boost (Figure 7g), reducing the size of the Trm pool to a level comparable to the Ad-NP control boost. Interestingly, Ad-NP boost was not affected by FTY720 treatment. Furthermore, FTY720 did not affect the frequency of NP-specific CD8 T cells in the lung. These data suggest that the enhancement of Trm cells in the lung through local 4-1BB stimulation relies on recruitment of additional antigen-specific cells from the circulation.

\section{The endogenous 4-1BB/4-1BBL pathway is critical for the formation or maintenance of the lung-resident CD8 population during primary influenza infection}

Having established that a supraphysiological dose of 4-1BBL can locally augment the pre-existing NP-specific Trm population in the lung, we next asked whether the endogenous 4-1BB costimulatory pathway also plays a role in the establishment of the lung Trm population during primary influenza infection. To test the role of endogenous 4-1BB signaling in Trm induction, we again used $4-1 \mathrm{BB}^{+1+}: 4-1 \mathrm{BB}^{-1-}$ mixed $\mathrm{BM}$ chimeras (Figure 8a). After reconstitution, mice were infected i.n. with a low dose of X31 and killed 1 month later to study lung-resident populations. Late after primary infection, the $\mathrm{NP}_{366-374}$-specific $\mathrm{CD} 8 \mathrm{~T}$ cells were almost exclusively from the $4-1 \mathrm{BB}^{+1+}$ donor. This dichotomy was not nearly as striking in the dLN or spleen (Figure $\mathbf{8 b}-\mathbf{d}$ ). Similar results were obtained for the immunodominant influenza $\mathrm{PA}_{224-233}$ epitope (data not shown). This endogenous $4-1 \mathrm{BB}^{+/+}$lung-resident memory pool comprised the conventional Trm-associated $\mathrm{CD}_{103}{ }^{+} \mathrm{CD} 9^{+}$as well as $\mathrm{CD} 103^{-} \mathrm{CD} 69^{+} \mathrm{T}$ cells
(Figure 8e). The lack of $4-1 \mathrm{BB}^{-1-}$ memory CD8 T cells specifically in the lung tissue suggests that the $4-1 \mathrm{BB} / 4-1 \mathrm{BBL}$ pathway acts directly on Trm and is critical for the establishment or maintenance of Trm during influenza infection.

\section{DISCUSSION}

Tissue-resident memory $\mathrm{T}$ cells provide a vital defense against infection at barrier and mucosal surfaces. ${ }^{6,7}$ Here we have shown that both supraphysiological as well as endogenous $4-1 \mathrm{BB}$ provide an indispensable signal for influenza virusspecific CD8 Trm. Using $4-1 \mathrm{BB}^{+/+}: 4-1 \mathrm{BB}^{-/-}$mixed BM chimeras, we show that there is an almost complete failure of $4-1 \mathrm{BB}^{-1-} \mathrm{T}$ cells to contribute to the lung-resident CD8 Trm pool after primary i.n. influenza infection. Moreover, administering 4-1BBL in a local boost with antigen in the same vector can induce a highly stable population of memory CD8 T cells that persists for almost a year and protects mice from lethal influenza challenge. This long-lived memory T-cell population includes a circulating effector memory subset that expresses higher levels of IL-7R compared with cells boosted without 4-1BBL as well as a robust Trm population, consisting of both $\mathrm{CD}_{103}{ }^{+}$and $\mathrm{CD}_{103}{ }^{-}$subsets. The accumulation of Trm in response to $4-1 \mathrm{BBL}$ is completely dependent on $4-1 \mathrm{BB}$ expression on the CD8 $\mathrm{T}$ cells (Figure 5), local delivery of $4-1 \mathrm{BBL}$ into the airways of the mouse (Figure $7 \mathbf{c}, \mathbf{d}$ ), recruitment of antigen-specific CD8 $\mathrm{T}$ cells from the circulation (Figure $7 \mathbf{e}-\mathbf{g}$ ), and inclusion of $\mathrm{Ag}$ and $4-1 \mathrm{BBL}$ in the same vector (data not shown).

The effect of local 4-1BBL administration on the Trm pool requires that new effector cells are recruited to the lung (Figure 7e-g). Previous work has shown that 4-1BB/4-1BBL interactions are dispensable for controlling the number of NP-specific CD8 T cells observed in the lung at days 9-10 of a primary infection with influenza $\mathrm{A} / \mathrm{X} 31$ as used here. ${ }^{35}$ Thus, it seems unlikely that $4-1 \mathrm{BBL}$ is involved in recruitment of the effectors to the lung per se, but rather in the establishment or maintenance of the Trm once these cells reach the tissue. Effects of exogenous 4-1BB stimulation on T-cell accumulation are largely due to effects on survival rather than on the initial rate of division. ${ }^{36}$ Consistently, we did not observe significant increases in bromodeoxyuridine uptake by $\mathrm{CD} 8 \mathrm{~T}$ cells boosted with or without 4-1BBL (data not shown). 4-1BB stimulation has been shown to impact multiple survival pathways, through NF- $\kappa B$-mediated upregulation of the anti-apoptotic genes $B c l-x L$ and Bfl1 (ref. 37) and TRAF1-Erk-dependent downmodulation of the proapoptotic Bim. ${ }^{36,38}$ Thus it is likely that 4-1BB mediates several pro-survival signals to enhance the Trm population. These survival signals may be important in keeping the antigen-specific $\mathrm{T}$ cells alive in the lung as they differentiate into Trm and/or they may be important in the maintenance of the already established Trm cells. Regardless, it is clear that without $4-1 \mathrm{BB}$, the Trm are virtually absent by day 30 post priming.

The effect of endogenous 4-1BB was much more striking on the lung Trm population, than the circulating effector memory population observed in the spleen at day 30 after primary 
a

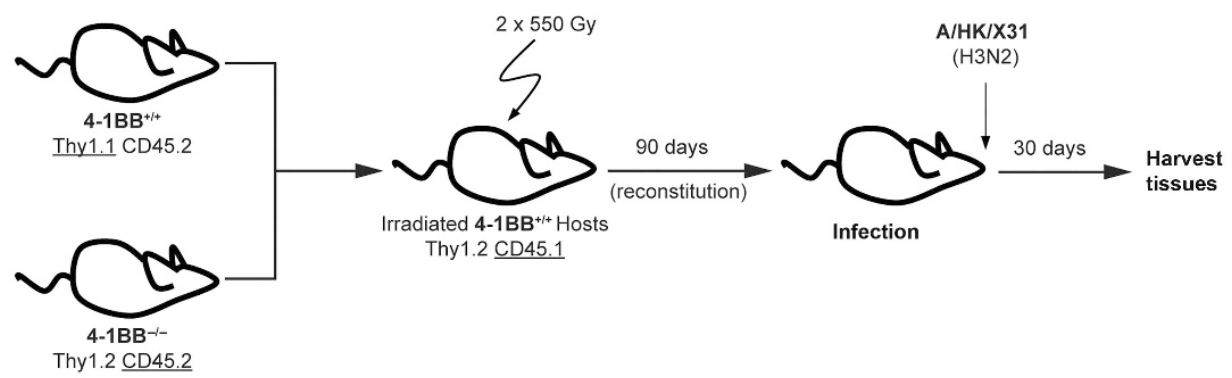

b

Day 30 post-X31 infection - Lung
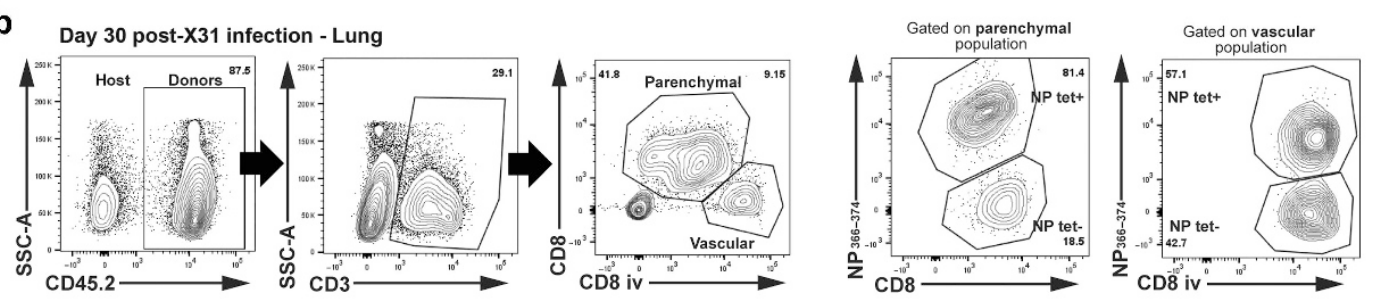

C

Day 30 post-X31 infection
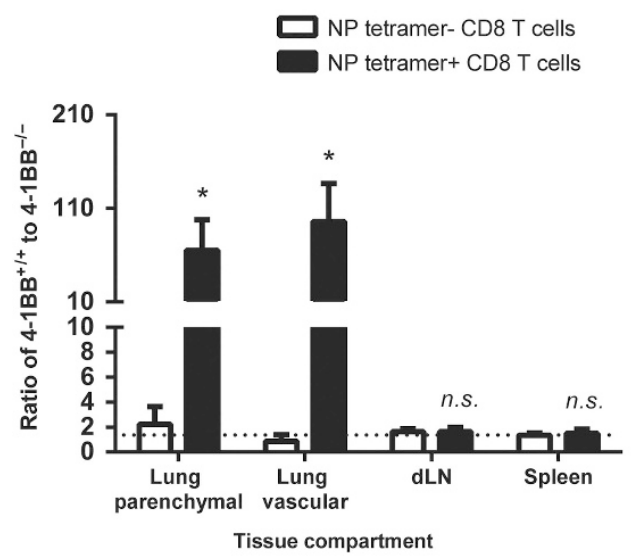

d

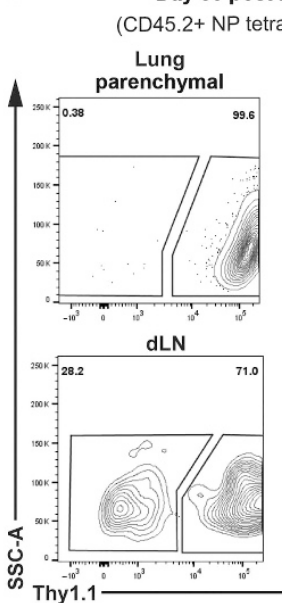

Day 30 post-X31 infection amer+ CD8 T cells)

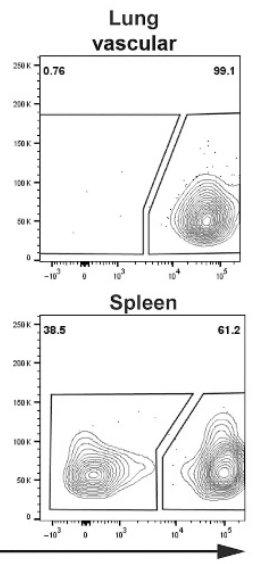

e

Lung parenchymal 4-1BB ${ }^{+++}$memory cells
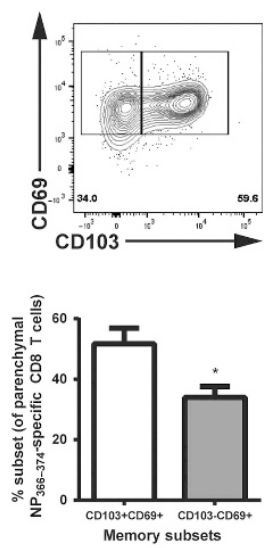

Figure 8 4-1BB expression on CD8 T cells is required for their contribution to the tissue-resident memory (Trm) compartment following primary influenza infection. (a) Mixed bone marrow (BM) chimeras were generated as described in Figure $\mathbf{5}$. After 90 days of reconstitution, chimeric mice were monitored for degree of chimerism in the blood and then infected i.n. with A/HK/X31 (5 HAU/mouse) and tissues were collected 30 days post-infection. (b) Representative gating for donor cells within the vascular and parenchymal memory CD8 T-cell compartments. (c) Ratios of CD45.2 donor 4-1BB ${ }^{+/+}$to $4-$ $1 \mathrm{BB}^{-1-}$ of $\mathrm{NP}_{366-374}$ tetramer-negative and $\mathrm{NP}_{366-374}$ tetramer-positive $\mathrm{CD} 8 \mathrm{~T}$ cells in the lung parenchyma, lung vasculature, draining lymph node (dLN), and spleen with (d) representative gating of the tetramer ${ }^{+}$population. Dotted line indicates pre-infection reconstitution ratio of CD8 T-cell compartment. Statistical analysis is shown between tetramer-negative and tetramer-positive CD8 T cells. (e) Frequency and representative gating for CD103 ${ }^{+}$CD69 ${ }^{+}$ and $\mathrm{CD} 103^{-} \mathrm{CD}^{-} 9^{+}$Trm populations within the lung parenchymal $\mathrm{NP}_{366-374^{-}-\text {specific CD8 } \mathrm{T} \text { cells from the 4-1BB }}{ }^{+/+}$compartment. Data are pooled from three separate experiments for a total of seven chimeric mice, with two-three mice per experiment (mean \pm s.e.m.). NS not significant, ${ }^{\star} P<0.05$ (Wilcoxon test for $\mathbf{c}$ or Mann-Whitney test for $\mathbf{e}$ ).

influenza infection. Previous work showed that 4-1BBL has about a 2 -fold effect on the Tem population observed in the spleen at day 38 post-prime. ${ }^{14}$ However, in the current studies, this small effect did not reach statistical significance (Figure 8c). In contrast, $4-1 \mathrm{BB}^{+/+}$cells almost exclusively populated the lung Trm pool (Figure 8c). On the other hand, when supraphysiological 4-1BBL signals are given in the boost using the Ad-NP-4-1BBL vector, effects on Tem and Trm were both dramatic. This is likely because $4-1 \mathrm{BB}$ is more highly expressed upon reactivation of primed $\mathrm{T}$ cells than on naive $\mathrm{T}$ cells ${ }^{16}$ and because the amount of endogenous $4-1 \mathrm{BBL}$ is much lower than the amount achieved with the adenovirus vector.
Adenoviruses can enter cells that express the coxsackievirus and adenovirus receptor, such as airway epithelial cells, ${ }^{39}$ but are also known to infect monocytes and dendritic cells by a coxsackievirus and adenovirus receptor-independent pathway. ${ }^{40,41}$ Previous work showed that Ad-4-1BBL induces 4-1BBL expression on both $\mathrm{CD} 45^{-}$and $\mathrm{CD} 45^{+}$cells in the lung, with the former showing greater expression ${ }^{35}$ and no detectable expression in the draining LN, suggesting a largely local effect. Given that the airway epithelial cells are by far the most abundant targets for adenoviral infection in the lung and the very low viral vector dose administered in our model, it is plausible that the NP and 4-1BBL delivered by the Ad vector are 
presented to the $\mathrm{T}$ cells by epithelial cells to induce local effects on the Trm or in the recruited Trm precursors. The ability of 4-1BBL to impact $\mathrm{T}$ cell responses through non-professional antigen presenting cells is perhaps not surprising given the CD28-independence of the $4-1 \mathrm{BB}$ signal. ${ }^{42}$ The source of endogenous 4-1BBL for driving expansion of the Trm population during primary influenza infection remains to be determined. However, 4-1BBL is expressed on $\mathrm{CD} 11 \mathrm{c}^{\text {hi }} \mathrm{CD} 11 \mathrm{~b}^{\text {hi }} \mathrm{MHC} \mathrm{II}{ }^{+}$cells in the lung of influenza infected mice ${ }^{43}$ and $4-1 \mathrm{BBL}$ mRNA is also present albeit at low levels in the lungs of unimmunized mice as measured by polymerase chain reaction. ${ }^{35}$

Although anti-4-1BB agonist antibodies have been administered systemically to induce expansion of T cells, ${ }^{44,45}$ this is associated with expansion of endogenous CD4 and CD8 effector memory cells independent of immunization ${ }^{46}$ and leads to immunopathology. ${ }^{20}$ In contrast, by delivering $4-1 \mathrm{BBL}$ in the same vector as antigen straight into the airways, we did not observe splenomegaly or increased liver enzyme activity that is observed with systemic anti-4-1BB administration. ${ }^{20}$ This is likely because the low-dose local delivery of 4-1BBL with antigen preferentially targets the antigen-specific response to the immunogen.

IL-7R has been identified as necessary but not sufficient for the formation of memory CD8 T cells in the context of viral infection. ${ }^{47}$ In the present study, we identified greater IL-7R expression, both in frequency at early time points and in per cell expression late in the response in 4-1BBL-boosted circulating and lung-resident memory CD8 T cells. IL-7R expression is correlated with the pro-survival molecule Bcl-2 and identifies long-lived memory precursor cells. ${ }^{23}$ However, it is difficult determine the importance of the relatively small increase in per cell level of expression of the IL-7 receptor for the 4-1BBLmediated effects, given that IL-7R is necessary for memory CD8 $\mathrm{T}$ cell formation in general during viral infection ${ }^{47}$ and blocking IL-7R during the boost similarly attenuated memory formation in both the Ad-NP and Ad-NP-4-1BBL groups. Antigen presentation can be detected within draining lymph nodes for at least 40 days post-immunization with replication-defective adenoviruses, with CD8 $\mathrm{T}$ cells exhibiting signs of chronic activation even out to 60 days. ${ }^{27}$ Thus the prolonged expression of NP and 4-1BBL in the airways may be an important component of the dramatically enlarged Trm population and the long-lived protective response. Indeed, prolonged antigen stimulation has been associated with Tem differentiation. ${ }^{48}$ Furthermore, prolonged cognate antigen stimulation has been implicated in maintaining the expression of the Trm marker CD103. ${ }^{8}$ Despite the reported persistence of Ad-induced antigen presentation for $\sim 2$ months, 4 -1BBL administration in the present study induces Trm for at least 210 days postboost and protection out to at least a year. It is possible that durable changes induced by $4-1 \mathrm{BBL}$ are either programmed during the period that the adenovirus delivered genes are expressed, or potentially that the initial response is maintained by the lower level of endogenously expressed $4-1 B B L .{ }^{35}$ Indeed, in earlier adoptive transfer studies of circulating CD8 T-cell memory, 4-1BBL was found to be dispensable for priming, but required for maintenance of CD8 memory T cells. ${ }^{18}$ It is also important to note that prolonged antigen-presentation by replication-defective adenovirus vectors at high doses can induce a CD8 T-cell phenotype consistent with partially exhausted Tem cells. ${ }^{27}$ This was observed in our study as well. At higher dose of Ad-NP, we saw a loss in the ability of memory CD8 $\mathrm{T}$ cells to produce multiple cytokines (Figure $\mathbf{3 d , e}$ ). However, boosting with $4-1 \mathrm{BBL}$ induced a polyfunctional influenza-specific CD8 T-cell response at a much lower dose of Ad vector and antigen, potentially avoiding the exhaustive effect of high-dose persisting antigen.

In sum, we have shown that $4-1 \mathrm{BB}$ is critical on CD8 T cells for induction of a long-lived lung Trm population through endogenous as well as supraphysiological stimulation. This signal must be locally delivered and is critical in the induction of optimal T-cell responses within the lung for protective immunity against influenza infection. Although the inclusion of a self-molecule like $4-1 \mathrm{BBL}$ may not be a practical vaccine strategy, the upregulation of $4-1 \mathrm{BBL}$ and other TNFR-family ligands is an important factor to consider when developing adjuvants for future vaccines.

\section{METHODS}

Mice. C57BL/6 mice were purchased from Charles River Laboratories (St. Constant, Quebec, Canada). Both male and female mice were used for experiments, but were age- and sex-matched within each experiment. For BM chimera experiments, Thy1.1 (B6.PL-Thy $\left.{ }^{\mathrm{a}} / \mathrm{CyJ}\right)$ and CD45.1 (C57Bl/6-Ly5.1) mice were purchased from Jackson Laboratories (Bar Harbor, USA), with the latter bred in-house at the University of Toronto. 4-1BB ${ }^{-1-}$ mice, bred on the C57BL/6 background were obtained from B. Kwon (Tulane University, New Orleans, LA) ${ }^{49}$ and used as source of donor cells for BM chimeras. All mice were housed in sterile micro-isolator cages under specific pathogen-free conditions. Animal studies were approved by the University of Toronto Animal Care Committee in accordance with the regulations of the Canadian Council on Animal Care.

Viruses. Influenza A/HK/X31 and A/PR8 viruses were grown in eggs and their tissue culture infectious dose determined by infection of MDCK cells. ${ }^{50}$ Mice (5-6 weeks of age) were immunized with $30 \mu \mathrm{l}$ of diluted virus at the indicated doses by i.n. infection while anaesthetized with isofluorane. For PR8 infections, mice were monitored closely with daily weights taken and were killed when moribund. Replicationdefective adenovirus 5 expressing influenza NP in the E1 region were kindly provided by J. Bramson (McMaster University, Hamilton, ON, Canada). The NP gene was derived from influenza A/PR8 GenBank: J02147.1. Adenovirus expressing NP in E3 and 4-1BBL in E1 were generated as previously published. ${ }^{15}$ Immunizations of mice with adenovirus were performed at the indicated doses and schedule by i.n. or i.p. injections as indicated.

Tissue harvest and processing. Mice were injected intravenously with $3 \mu \mathrm{g}$ of anti-mouse $\mathrm{CD} 8 \alpha$ (clone: 53-6.7) antibody conjugated to PE (eBioscience, San Diego, CA) or BV605 (BioLegend, San Diego, $\mathrm{CA}$ ) and killed 10 min later. ${ }^{28}$ Lung tissue was perfused with $10 \mathrm{ml}$ of PBS to remove cells in the pulmonary vasculature, and then minced and digested in 100 units per $\mathrm{ml}$ of collagenase IV (Invitrogen, Carlsbad, CA) for $1 \mathrm{~h}$ at $37^{\circ}$ in a shaker. Spleen (after RBC lysis) and dLN were mechanically disrupted and filtered through a $70 \mu \mathrm{M}$ to create single cell suspensions. Blood was collected from the saphenous vein and treated with RBC lysis buffer. 
Flow cytometry and intracellular cytokine staining. Influenza $\mathrm{NP}_{366-374}$-specific CD8 $\mathrm{T}$ cells were analyzed using MHC class I tetramers obtained from the National Institute for Allergy and Infectious Disease tetramer facility (Emory University, Atlanta, GA). For intracellular cytokine staining, lung and spleen samples were restimulated ex vivo with $1 \mu \mathrm{M}$ of the $\mathrm{MHC}$ I-restricted $\mathrm{NP}_{366-74}$ peptide for $6 \mathrm{~h}$ with GolgiStop (BD Pharmingen, San Jose, CA) at $37^{\circ} \mathrm{C}$. Cells were surfaced stained, fixed, permeabilized, and stained intracellularly for the appropriate cytokines. Unstimulated samples (no peptide) were used as negative controls. Samples were analyzed using LSRFortessa (BD Biosciences), and FlowJo (TreeStar, Ashland, OR) software.

Antibodies. The antibodies used in this study are as follows: antimouse CD $8 \alpha$ (clone: 53-6.7) (eBioscience), anti-mouse CD3 $\varepsilon$ (clone: 145-2C11) (eBioscience, BioLegend), anti-mouse Thy1.1 (clone: HIS51) (eBioscience), anti-mouse CD62L (clone: MEL-14) (eBioscience), anti-mouse CD127 (clone: A7R34) (eBioscience), antimouse IFN $\gamma$ (clone: XMG1.2) (eBioscience), anti-mouse TNF $\alpha$ (clone: MP6-XT22) (BD Pharmingen), anti-mouse IL-2 (clone: JES6-5H4) (eBioscience), anti-mouse CD107a (clone: 1D4B) (eBioscience), antimouse T-bet (clone: 4B10) (eBioscience), anti-mouse Eomesodermin (clone: Dan11mag) (eBioscience), rabbit anti-mouse TCF-1 (clone: MA5-14965) from Thermo Fisher (Waltham, MA) with labeled secondary donkey anti-rabbit (Thermo Fisher), anti-mouse CD69 (clone: H1.2F3) (eBioscience), anti-mouse CD103 (clone: 2E7) (eBioscience), anti-mouse CD49a (clone: Ha31/8) (BD Pharmingen), and fixable viability dye (eBioscience). In vivo IL-7R blockade was performed with i.p. injections of $400 \mu \mathrm{g}$ per dose per mouse of antiCD127 monoclonal antibody (clone: A7R34) at two doses, 2 days apart in the indicated immunization schedule. The hybridoma was kindly provided by C. Paige (University Health Network, Toronto, ON, Canada).

Mixed bone marrow chimeras. Bone marrow was obtained from the femur and tibia of wildtype Thy $1.1^{+1+} \mathrm{CD} 45.2^{+1+} 4-1 \mathrm{BB}+1+$ and Thy $1.2^{+1+} \mathrm{CD} 45.2^{+1+} 4-1 \mathrm{BB}^{-1-}$ mice and transferred at a $1: 1$ ratio into host Thy1.2 $2^{+/+} \mathrm{CD} 45.1^{+/+} 4-1 \mathrm{BB}^{+/+}$mice that were lethally irradiated with two doses of $550 \mathrm{rad} .2 .5 \times 10^{6}$ cells of each donor type were mixed and transferred in a total of $200 \mu \mathrm{l}$ by intravenous tail vein injection. Reconstituted chimeric mice received water supplemented with $2 \mathrm{mg} \mathrm{ml}^{-1}$ neomycin sulfate (Bio-Shop, Burlington, ON, Canada) during the first 2 weeks, and were rested for a total of 90 days before chimerism was checked. They were then immunized according to the indicated schedules.

FTY720 treatment. Mice were given daily a $200 \mu$ of FTY720 (SigmaAldrich, St Louis, MO) by i.p. injection at a dose of $1 \mathrm{mg} \mathrm{kg}^{-1}$ diluted in PBS, according to the indicated treatment regimen.

Statistics. Statics were performed using GraphPad Prism 6 (San Diego, CA), with the specific test performed as indicated in the figure legends.

\section{ACKNOWLEDGMENTS}

We thank Byoung Kwon for 4-1BB ${ }^{-1-}$ mice, Tao Wen for generation of the Ad-NP-4-1BBL vector, and Birinder Ghumman for production and titering of adenovirus and influenza virus stocks. Funded by Canadian Institutes of Health Research (CIHR) grants MOP-133443 and FDN-143250 to T.H.W. THW holds the Sanofi Pasteur Chair in Human Immunology at the University of Toronto. A.C.Z. was funded by a Queen Elizabeth II/Aventis Graduate Scholarship, Ontario Graduate Scholarship (OGS) and a CIHR Doctoral Award.

\section{AUTHOR CONTRIBUTIONS}

A.C.Z. and T.H.W. designed the study, analyzed the data, and wrote the manuscript. A.C.Z. performed all experiments with technical assistance from L.E.W. and M.E.W. on the long-term blood-tracking cohorts.

\section{DISCLOSURE}

The authors declare no conflict of interest.

c) 2017 Society for Mucosal Immunology

\section{REFERENCES}

1. Krammer, F. \& Palese, P. Advances in the development of influenza virus vaccines. Nat. Rev. Drug Discov. 14, 167-182 (2015).

2. Grebe, K.M., Yewdell, J.W. \& Bennink, J.R. Heterosubtypic immunity to influenza A virus: where do we stand?. Microbes Infect. 10, 1024-1029 (2008).

3. Hillaire, M.L. et al. Characterization of the human CD8 T cell response following infection with 2009 pandemic influenza H1N1 virus. J. Virol. 85, 12057-12061 (2011).

4. Wagar, L.E. et al. Humoral and cell-mediated immunity to pandemic H1N1 influenza in a Canadian cohort one year post-pandemic: implications for vaccination. Plos One 6, e28063 (2011).

5. McElhaney, J.E. et al. The unmet need in the elderly: how immunosenescence, CMV infection, co-morbidities and frailty are a challenge for the development of more effective influenza vaccines. Vaccine 30, 2060-2067 (2012).

6. Schenkel, J.M. \& Masopust, D. Tissue-resident memory T cells. Immunity 41, 886-897 (2014)

7. Mueller, S.N. \& Mackay, L.K. Tissue-resident memory T cells: local specialists in immune defence. Nat. Rev. Immunol. 16, 79-89 (2016).

8. Wu, T. et al. Lung-resident memory CD8 T cells (TRM) are indispensable for optimal cross-protection against pulmonary virus infection. J. Leukoc. Biol. 95, 215-224 (2014).

9. McMaster, S.R., Wilson, J.J., Wang, H. \& Kohlmeier, J.E. Airway-resident memory cd8 t cells provide antigen-specific protection against respiratory virus challenge through rapid IFN- $\gamma$ production. J. Immunol. 195, 203-209 (2015).

10. Hogan, R.J. et al. Activated antigen-specific CD8 + T cells persist in the lungs following recovery from respiratory virus infections. J. Immunol. 166, 1813-1822 (2001).

11. Liang, S., Mozdzanowska, K., Palladino, G. \& Gerhard, W. Heterosubtypic immunity to influenza type A virus in mice. Effector mechanisms and their longevity. J. Immunol. 152, 1653-1661 (1994).

12. Chen, L. \& Flies, D.B. Molecular mechanisms of T cell co-stimulation and co-inhibition. Nat. Rev. Immunol. 13, 227-242 (2013).

13. DeBenedette, M.A. et al. Analysis of 4-1BB ligand (4-1BBL)-deficient mice and of mice lacking both 4-1BBL and CD28 reveals a role for 4-1BBL in skin allograft rejection and in the cytotoxic $T$ cell response to influenza virus. J. Immunol. 163, 4833-4841 (1999).

14. Bertram, E.M., Lau, P. \& Watts, T.H. Temporal segregation of 4-1BB versus CD28-mediated costimulation: 4-1BB ligand influences Tcell numbers late in the primary response and regulates the size of the T cell memory response following influenza infection. J. Immunol. 168, 3777-3785 (2002).

15. Moraes, T.J., Lin, G.H., Wen, T. \& Watts, T.H. Incorporation of 4-1BB ligand into an adenovirus vaccine vector increases the number of functional antigen-specific CD8 T cells and enhances the duration of protection against influenza-induced respiratory disease. Vaccine 29, 6301-6312 (2011)

16. Lin, G.H. et al. Evaluating the cellular targets of anti-4-1BB agonist antibody during immunotherapy of a pre-established tumor in mice. PLoS One 5, e11003 (2010).

17. Harrison, J.M. et al. 4-1BBL coexpression enhances HIV-specific CD8 T cell memory in a poxvirus prime-boost vaccine. Vaccine $24,6867-6874$ (2006).

18. Bertram, E.M. et al. A switch in costimulation from CD28 to 4-1BB during primary versus secondary CD8 $\mathrm{T}$ cell response to influenza in vivo. J. Immunol. 172, 981-988 (2004).

19. Thomas, P.G., Keating, R., Hulse-Post, D.J. \& Doherty, P.C. Cell-mediated protection in influenza infection. Emerg. Infect. Dis. 12, 48-54 (2006).

20. Niu, L. et al. Cytokine-mediated disruption of lymphocyte trafficking, hemopoiesis, and induction of Iymphopenia, anemia, and thrombocytopenia in anti-CD137-treated mice. J. Immunol. 178, 4194-4213 (2007). 
21. Harty, J.T. \& Badovinac, V.P. Shaping and reshaping CD8 + T-cell memory. Nat. Rev. Immunol. 8, 107-119 (2008).

22. Sallusto, F., Lenig, D., Forster, R., Lipp, M. \& Lanzavecchia, A. Two subsets of memory $\mathrm{T}$ lymphocytes with distinct homing potentials and effector functions. Nature 401, 708-712 (1999).

23. Kaech, S.M. et al. Selective expression of the interleukin 7 receptor identifies effector CD8 T cells that give rise to long-lived memory cells. Nat. Immunol. 4, 1191-1198 (2003).

24. Bachmann, M.F., Wolint, P., Schwarz, K., Jäger, P. \& Oxenius, A. Functional properties and lineage relationship of $\mathrm{CD} 8^{+} \mathrm{T}$ cell subsets identified by expression of IL-7 receptor alpha and CD62L. J. Immunol. 175, 46864696 (2005).

25. Kaech, S.M. \& Cui, W. Transcriptional control of effector and memory $\mathrm{CD}^{+}$T cell differentiation. Nat. Rev. Immunol. 12, 749-761 (2012).

26. Zhou, X. et al. Differentiation and persistence of memory CD8(+) T cells depend on T cell factor 1. Immunity 33, 229-240 (2010).

27. Yang, T.C. et al. The $\mathrm{CD}^{+} \mathrm{T}$ cell population elicited by recombinant adenovirus displays a novel partially exhausted phenotype associated with prolonged antigen presentation that nonetheless provides long-term immunity. J. Immunol. 176, 200-210 (2006).

28. Anderson, K.G. et al. Cutting edge: intravascular staining redefines lung CD8 T cell responses. J. Immunol. 189, 2702-2706 (2012).

29. Lee, Y.T. et al. Environmental and antigen receptor-derived signals support sustained surveillance of the lungs by pathogen-specific cytotoxic $T$ lymphocytes. J. Virol. 85, 4085-4094 (2011).

30. Mackay, L.K. et al. T-box transcription factors combine with the cytokines TGF- $\beta$ and IL-15 to control tissue-resident memory Tcell fate. Immunity 43, 1101-1111 (2015).

31. Shen, C.H. et al. Loss of IL-7R and IL-15R expression is associated with disappearance of memory T cells in respiratory tract following influenza infection. J. Immunol. 180, 171-178 (2008).

32. Kohlmeier, J.E., Miller, S.C. \& Woodland, D.L. Cutting edge: antigen is not required for the activation and maintenance of virus-specific memory CD8 + T cells in the lung airways. J. Immunol. 178, 4721-4725 (2007).

33. Ray, S.J. et al. The collagen binding alpha1beta1 integrin VLA-1 regulates CD8 T cell-mediated immune protection against heterologous influenza infection. Immunity 20, 167-179 (2004).

34. Matloubian, M. et al. Lymphocyte egress from thymus and peripheral lymphoid organs is dependent on S1P receptor 1 . Nature 427, 355-360 (2004).

35. Lin, G.H. et al. Endogenous 4-1BB ligand plays a critical role in protection from influenza-induced disease. J. Immunol. 182, 934-947 (2009).
36. Sabbagh, L. et al. A critical role for TNF receptor-associated factor 1 and Bim down-regulation in CD8 memory Tcell survival. Proc. Natl. Acad. Sci. USA 103, 18703-18708 (2006).

37. Lee, H.W. et al. 4-1BB promotes the survival of CD8(+) T lymphocytes by increasing expression of $\mathrm{Bcl}-\mathrm{x}(\mathrm{L})$ and $\mathrm{Bfl}-1$. J. Immunol. 169, 4882-4888 (2002).

38. Sabbagh, L., Pulle, G., Liu, Y., Tsitsikov, E.N. \& Watts, T.H. ERK-dependent bim modulation downstream of the 4-1BB-TRAF1 signaling axis is a critical mediator of CD8 Tcell survival in vivo. J. Immunol. 180, 8093-8101 (2008).

39. Bergelson, J.M. et al. Isolation of a common receptor for Coxsackie B viruses and adenoviruses 2 and 5. Science 275, 1320-1323 (1997).

40. Loré, K. et al. Myeloid and plasmacytoid dendritic cells are susceptible to recombinant adenovirus vectors and stimulate polyfunctional memory $T$ cell responses. J. Immunol. 179, 1721-1729 (2007).

41. Rozis, G. et al. Langerhans cells are more efficiently transduced than dermal dendritic cells by adenovirus vectors expressing either group $\mathrm{C}$ or group B fibre protein: implications for mucosal vaccines. Eur. J. Immunol. 35, 2617-2626 (2005).

42. DeBenedette, M.A., Shahinian, A., Mak, T.W. \& Watts, T.H. Costimulation of CD28- T lymphocytes by 4-1BB ligand. J. Immunol. 158, 551-559 (1997).

43. Snell, L.M., Lin, G.H., McPherson, A.J., Moraes, T.J. \& Watts, T.H. T-cell intrinsic effects of GITR and 4-1BB during viral infection and cancer immunotherapy. Immunol. Rev. 244, 197-217 (2011).

44. Shuford, W.W. et al. 4-1BB costimulatory signals preferentially induce CD8 + Tcell proliferation and lead to the amplification in vivo of cytotoxic $T$ cell responses. J. Exp. Med. 186, 47-55 (1997).

45. Takahashi, C., Mittler, R.S. \& Vella, A.T. Cutting edge: 4-1BB is a bona fide CD8 T cell survival signal. J. Immunol. 162, 5037-5040 (1999).

46. Zhu, Y., Zhu, G., Luo, L., Flies, A.S. \& Chen, L. CD137 stimulation delivers an antigen-independent growth signal for $\mathrm{T}$ lymphocytes with memory phenotype. Blood 109, 4882-4889 (2007).

47. Hand, T.W., Morre, M. \& Kaech, S.M. Expression of IL-7 receptor alpha is necessary but not sufficient for the formation of memory CD8 Tcells during viral infection. Proc. Natl. Acad. Sci. USA 104, 11730-11735 (2007).

48. Lanzavecchia, A. \& Sallusto, F. Understanding the generation and function of memory T cell subsets. Curr. Opin. Immunol. 17, 326-332 (2005).

49. Kwon, B.S. et al. Immune responses in 4-1BB (CD137)-deficient mice. J. Immunol. 168, 5483-5490 (2002).

50. Cottey, R., Rowe, C.A. \& Bender, B.S. Influenza virus. Curr. Protoc. Immunol. Chapter 19, Unit 19.11 (2001). 